\title{
A Binary Intuitionistic Fuzzy Relation: Some New Results, a General Factorization, and Two Properties of Strict Components
}

\author{
Louis Aimé Fono, ${ }^{1}$ Gilbert Njanpong Nana, ${ }^{2}$ Maurice Salles, ${ }^{3}$ \\ and Henri Gwet ${ }^{4}$ \\ ${ }^{1}$ Département de Mathématiques et Informatique, Faculté des Sciences, Université de Douala, \\ B.P. 24157 Douala, Cameroon \\ ${ }^{2}$ Laboratoire de Mathématiques Appliquées aux Sciences Sociales, Département de Mathématiques, \\ Faculté des Sciences, Université de Yaoundé I, B.P. 15396 Yaoundé, Cameroon \\ ${ }^{3}$ MRSH, University of Caen, CREM-UMR 6211, CNRS, 14032 Caen Cedex, France \\ ${ }^{4}$ Department of Mathematics, National Polytechnic Institute, P.O. Box 8390, Yaoundé, Cameroon
}

Correspondence should be addressed to Louis Aimé Fono, lfono2000@yahoo.fr

Received 3 July 2008; Revised 24 December 2008; Accepted 15 June 2009

Recommended by Andrzej Skowron

\begin{abstract}
We establish, by means of a large class of continuous t-representable intuitionistic fuzzy t-conorms, a factorization of an intuitionistic fuzzy relation (IFR) into a unique indifference component and a family of regular strict components. This result generalizes a previous factorization obtained by Dimitrov (2002) with the ( $\max , \min$ ) intuitionistic fuzzy t-conorm. We provide, for a continuous $\mathrm{t}$-representable intuitionistic fuzzy t-norm $\tau$, a characterization of the $\tau$-transitivity of an IFR. This enables us to determine necessary and sufficient conditions on a $\tau$-transitive IFR $R$ under which a strict component of $R$ satisfies pos-transitivity and negative transitivity.
\end{abstract}

Copyright (C) 2009 Louis Aimé Fono et al. This is an open access article distributed under the Creative Commons Attribution License, which permits unrestricted use, distribution, and reproduction in any medium, provided the original work is properly cited.

\section{Introduction}

In the real life, individual or collective preferences are not always crisp; they can be also ambiguous. Since 1965 when Zadeh [1] introduced fuzzy set theory, researchers [2-11] modelled such preferences by (binary) fuzzy relation (simply denoted by FR) on $X$, that is, a function $R: X \times X \rightarrow[0,1]$ where $X$ is a set of alternatives with $\operatorname{Card}(X)=|X| \geq 3$. In this case, for $(x, y) \in X^{2}, R(x, y)$ is interpreted as the degree to which $x$ is "at least as good as" $y$. If $\forall x, y \in X, R(x, y) \in\{0,1\}$, then $R$ is crisp, and we denote $R(x, y)=1$ by $x R y$ and $R(x, y)=0$ by $\operatorname{not}(x R y)$. Literature on the theory of fuzzy relations and on applications of fuzzy relations in other fields such as economics and in particular social choice theory is growing. 
Since 1983 when Atanassov $[12,13]$ introduced intuitionistic fuzzy sets (IFSs), some scholars [14-19] modelled ambiguous preferences by a (binary) intuitionistic fuzzy relation (IFR) on $X$, that is, a function $R: X \times X \rightarrow L^{*}=\left\{\left(a_{1}, a_{2}\right) \in[0,1]^{2}, a_{1}+a_{2} \leq 1\right\}$ where $\forall x, y \in X, R(x, y)=\left(\mu_{R}(x, y), v_{R}(x, y)\right)$. In this case, $\mu_{R}(x, y)$ is the degree to which $x$ is "at least as good as" $y$, and $v_{R}(x, y)$ is the degree to which $x$ is not "at least as good as" $y$. The positive real number $\pi_{R}(x, y)=1-\mu_{R}(x, y)-v_{R}(x, y)$ (since $\mu_{R}(x, y)+v_{R}(x, y) \leq 1$ ), usually called fuzzy index, indicates the degree of incomparability between $x$ and $y$. In this paper, we simply write $\forall x, y \in X, R(x, y)=\left(\mu_{R}(x, y), v_{R}(x, y)\right)$. Clearly, we have two particular cases: (i) if $\forall(x, y) \in X \times X, \pi_{R}(x, y)=0$, that is, $v_{R}(x, y)=1-\mu_{R}(x, y)$, then $R$ becomes an FR on $X$, and (ii) if $\forall(x, y) \in X \times X, \pi_{R}(x, y)=0$ and $v_{R}(x, y) \in\{0,1\}$, then $R$ becomes the well-known (binary) crisp relation. In the first case, we simply write $R(x, y)=\mu_{R}(x, y)$, and in the second case, we have $x R y \Leftrightarrow \mu_{R}(x, y)=1$ (i.e., $v_{R}(x, y)=0$ ).

A factorization of a binary relation is an important question in preference modelling. In that view, Dimitrov [18] established a factorization of an IFR into an indifference and a strict component in the particular case where the union is defined by means of the (max, $\min$ ) t-representable intuitionistic fuzzy t-conorm. Recently, Cornelis et al. [20] established some results on t-representable intuitionistic fuzzy t-norms (i.e., $\tau=(T, S)$ where $S$ is a fuzzy $\mathrm{t}$ conorm, and $T$ is a fuzzy t-norm satisfying $\forall a, b \in[0,1], T(a, b) \leq 1-S(1-a, 1-b))$, on t-representable intuitionistic fuzzy t-conorms (i.e., $\partial=(S, T)$ ) and on intuitionistic fuzzy implications. Thereby, our goal is to generalize Dimitrov's framework [18] and to establish some results on IFRs by means of continuous t-representable intuitionistic fuzzy t-norms and t-conorms.

The aim of this paper is (i) to study the standard completeness of an IFR, (ii) to establish a characterization of the $\tau$-transitivity of an IFR, (iii) to generalize the factorization of an IFR established by Dimitrov [18], and (iv) to determine necessary and sufficient conditions on a $\tau$-transitive IFR $R$ under which a given strict component of $R$ (obtained in our factorization) satisfies respectively pos-transitivity and negative transitivity.

First we establish some useful results on t-representable intuitionistic fuzzy t-norms, t-representable intuitionistic fuzzy t-conorms, and intuitionistic fuzzy implications.

The paper is organized as follows. In Section 2, we recall some basic notions and properties on fuzzy operators and intuitionistic fuzzy operators which we need throughout the paper. We also establish some useful results on fuzzy implications and intuitionistic fuzzy implications. Section 3 has three subsections. In Section 3.1, we recall some basic and useful definitions on IFRs. In Section 3.2, we introduce the standard completeness, namely, a $(S, T)$-completeness of an IFR. We make clear that the notion of completeness introduced by Dimitrov [18] is not standard, but it is weaker than a standard one. In Section 3.3, we establish, for a given $\tau$, a characterization of the $\tau$-transitivity of an IFR. Section 4 is devoted to a new factorization of an IFR, and it has two subsections. In Section 4.1, we recall the factorization of an IFR established by Dimitrov [18] with the (max, min) intuitionistic fuzzy t-conorm. We point out some intuitive difficulties of the strict component obtained in [18]. In Section 4.2, we introduce definitions of an indifference and a strict component of an IFR, and we establish a general factorization of an IFR for a large class of continuous t-representable intuitionistic fuzzy t-conorms. Section 5 contains two subsections. In Section 5.1, we introduce intuitionistic fuzzy counterparts of pos-transitivity and negative transitivity of a crisp relation. We justify that there exists some IFRs (noncrisp and non FRs) which violate each of these two properties. This forces us in Section 5.2 to establish necessary and sufficient conditions on a $\tau$-transitive IFR $R$, such that a strict component of $R$ satisfies, respectively, pos-transitivity and negative transitivity. Section 6 contains some 
concluding remarks. The proofs of our results are in the Appendix. (This was suggested by an anonymous referee.)

\section{Preliminaries on Operators}

Let $\leq_{L^{*}}$ be an order in $L^{*}$ defined by $\forall\left(a_{1}, a_{2}\right),\left(b_{1}, b_{2}\right) \in L^{*},\left(a_{1}, a_{2}\right) \leq_{L^{*}}\left(b_{1}, b_{2}\right) \Leftrightarrow\left(a_{1} \leq b_{1}\right.$ and $\left.a_{2} \geq b_{2}\right) .\left(L^{*}, L_{L^{*}}\right)$ is a complete lattice. $0_{L^{*}}=(0,1)$ and $1_{L^{*}}=(1,0)$ are the units of $L^{*}$.

In the following section, we recall some definitions, examples, and well-known results on fuzzy t-norms, fuzzy t-conorms, fuzzy implications, and fuzzy coimplicators.

\subsection{Review on Fuzzy Operators}

We firstly recall notions on fuzzy t-norms and fuzzy t-conorms (see [21, 22]).

A fuzzy t-norm (resp. a fuzzy t-conorm) is an increasing, commutative, and associative binary operation on $[0,1]$ with a neutral 1 (resp. 0 ). The dual of a fuzzy t-norm $T$ is a fuzzy $\mathrm{t}$-conorm $S$, that is, $\forall a, b \in[0,1], T(a, b)=1-S(1-a, 1-b)$.

Let us recall two usual families of fuzzy t-norms and fuzzy t-conorms. The Frank $\mathrm{t}$ norms $\left(T_{F}^{l}\right)_{l \in[0, \infty]}$, that is, $\forall l \in[0, \infty], \forall a, b \in[0,1]$,

$$
T_{F}^{l}(a, b)= \begin{cases}T_{\mathbf{M}}(a, b)=\min (a, b)=a \wedge b, & \text { if } l=0, \\ T_{\mathbf{P}}(a, b)=a \times b, & \text { if } l=1, \\ T_{\mathbf{L}}(a, b)=\max (a+b-1,0), & \text { if } l=+\infty \\ \log _{l}\left(1+\frac{\left(l^{a}-1\right)\left(l^{b}-1\right)}{l-1}\right), & \text { otherwise }\end{cases}
$$

where $T_{\mathbf{M}}, T_{\mathbf{P}}$, and $T_{\mathbf{L}}$ are the minimum fuzzy t-norm, the product fuzzy t-norm, and the Łukasiewicz fuzzy t-norm, respectively. The Frank t-conorms $\left(S_{F}^{l}\right)_{l \in[0, \infty]}$, that is, $\forall l \in$ $[0, \infty], \forall a, b \in[0,1]$,

$$
S_{F}^{l}(a, b)= \begin{cases}S_{\mathbf{M}}(a, b)=\max (a, b)=a \vee b, & \text { if } l=0, \\ S_{\mathbf{P}}(a, b)=a+b-a \times b, & \text { if } l=1, \\ S_{\mathbf{L}}(a, b)=\min (a+b, 1), & \text { if } l=+\infty \\ 1-\log _{l}\left(1+\frac{\left(l^{1-a}-1\right)\left(l^{1-b}-1\right)}{l-1}\right), & \text { otherwise, }\end{cases}
$$

where $S_{\mathbf{M}}, S_{\mathbf{P}}$, and $S_{\mathbf{L}}$ are the maximum fuzzy $\mathrm{t}$-conorm, the product fuzzy $\mathrm{t}$-conorm, and the Łukasiewicz fuzzy t-conorm, respectively.

A fuzzy t-norm $T$ (fuzzy t-conorm $S$ ) is strict if $\forall a, b \in[0,1], \forall c \in] 0,1], a<b$ implies $T(a, c)<T(b, c)$ (resp. $\forall a, b \in[0,1], \forall c \in[0,1[, a<b$ implies $S(a, c)<S(b, c)$ ). The product fuzzy t-norm (resp. the product fuzzy t-conorm) is an example of a strict fuzzy t-norm (resp. fuzzy t-conorm). 
We have the following properties:

$$
\forall a, b \in[0,1], \quad\left\{\begin{array}{l}
\text { (i) } T(a, b) \leq \min (a, b) \\
\text { (ii) } \max (a, b) \leq S(a, b) .
\end{array}\right.
$$

Throughout the paper, $T$ is a continuous fuzzy t-norm, and $S$ is a continuous fuzzy t-conorm.

In the following, we recall some definitions and examples on fuzzy implications and fuzzy coimplicators based on fuzzy t-norms and fuzzy t-conorms, respectively (see [21-23]).

The fuzzy R-implication $I_{T}$ associated to $T$ is a binary operation on $[0,1]$ defined by $\forall a, b \in[0,1], I_{T}(a, b)=\max \{t \in[0,1], T(a, t) \leq b\}$. The fuzzy coimplicator $J_{S}$ associated to $S$ is a binary operation on $[0,1]$ defined by $\forall a, b \in[0,1], J_{S}(a, b)=\min \{t \in[0,1], b \leq S(a, t)\}$.

Let us recall some usual examples of these fuzzy operators.

The fuzzy R-implication associated to $T_{\mathbf{M}}$ is defined by

$$
\forall a, b \in[0,1], \quad I_{T_{\mathrm{M}}}= \begin{cases}1, & \text { if } a \leq b \\ b, & \text { if } a>b\end{cases}
$$

The fuzzy coimplicator associated to $S_{\mathbf{M}}$ is defined by

$$
\forall a, b \in[0,1], \quad J_{S_{\mathrm{M}}}(a, b)= \begin{cases}b, & \text { if } a<b, \\ 0, & \text { if } a \geq b\end{cases}
$$

The fuzzy R-implication associated to $T_{\mathrm{L}}$ is defined by

$$
\forall a, b \in[0,1], \quad I_{T_{\mathrm{L}}}(a, b)= \begin{cases}1, & \text { if } a \leq b, \\ 1-a+b, & \text { if } a>b .\end{cases}
$$

The fuzzy coimplicator associated to $S_{\mathrm{L}}$ is defined by

$$
\forall a, b \in[0,1], \quad J_{S_{\mathrm{L}}}(a, b)= \begin{cases}b-a, & \text { if } a<b, \\ 0, & \text { if } a \geq b .\end{cases}
$$

The fuzzy R-implication associated to $T_{P}$ is defined by

$$
\forall a, b \in[0,1], \quad I_{T_{\mathrm{P}}}(a, b)= \begin{cases}1, & \text { if } a \leq b \\ \frac{b}{a}, & \text { if } a>b\end{cases}
$$


The fuzzy coimplicator associated to $S_{P}$ is defined by

$$
\forall a, b \in[0,1], \quad J_{S_{\mathbf{p}}}(a, b)= \begin{cases}\frac{b-a}{1-a}, & \text { if } a<b, \\ 0, & \text { if } a \geq b .\end{cases}
$$

We complete the previous examples by giving expressions of fuzzy R-implications of the other Frank fuzzy t-norms and fuzzy coimplicators of the other Frank fuzzy t-conorms:

$$
\begin{gathered}
\forall l \in] 0,1[\cup] 1,+\infty\left[, \forall a, b \in[0,1], \quad I_{T_{F}^{l}}(a, b)= \begin{cases}1, & \text { if } a \leq b, \\
\log _{l}\left(1+\frac{(l-1)\left(l^{b}-1\right)}{l^{a}-1}\right), & \text { if } a>b,\end{cases} \right. \\
J_{S_{F}^{l}}(a, b)= \begin{cases}0, & \text { if } a \geq b, \\
1-\log _{l}\left(1+\frac{(l-1)\left(l^{1-b}-1\right)}{l^{(1-a)}-1}\right), & \text { if } a<b .\end{cases}
\end{gathered}
$$

We recall some useful properties on fuzzy implications and fuzzy coimplicators.

Proposition 2.1 (See $[4,5,9,21,23])$. For all $a, b, c \in[0,1]$,

(1) $I_{T}(a, a)=1 ; J_{S}(a, a)=0$, and $J_{S}(a, b) \leq b \leq I_{T}(a, b)$;

(2) $T\left(a, I_{T}(a, b)\right)=\min (a, b)$, and $S\left(a, J_{S}(a, b)\right)=\max (a, b)$;

(3)

$$
\begin{aligned}
& b<a \Longleftrightarrow I_{T}(a, b)<1, \\
& a<b \Longleftrightarrow J_{S}(a, b)>0 ;
\end{aligned}
$$

(4)

$$
a \leq b \Longrightarrow\left\{\begin{array}{l}
I_{T}(b, c) \leq I_{T}(a, c), \\
I_{T}(c, a) \leq I_{T}(c, b)
\end{array}\right.
$$

(5)

$$
a \leq b \Longrightarrow\left\{\begin{array}{l}
J_{S}(b, c) \leq J_{S}(a, c), \\
J_{S}(c, a) \leq J_{S}(c, b)
\end{array}\right.
$$

In the following, we recall some useful definitions and results on intuitionistic fuzzy operators. 


\subsection{Review on Intuitionistic Fuzzy Operators}

Definition 2.2 (See [20]). (1) An intuitionistic fuzzy t-norm is an increasing, commutative, and associative binary operation $\tau$ on $L^{*}$ satisfying $\forall(a, b) \in L^{*}, \tau((a, b),(1,0))=(a, b)$.

(2) An intuitionistic fuzzy t-conorm is an increasing, commutative, associative binary operation 2 on $L^{*}$ satisfying $\forall(a, b) \in L^{*}, \partial((a, b),(0,1))=(a, b)$.

Cornelis et al. [20] introduced an important class of intuitionistic fuzzy t-norms (resp. $\mathrm{t}$-conorms) based on fuzzy t-norms (resp. fuzzy t-conorms).

Definition 2.3. An intuitionistic fuzzy t-norm $\tau$ (resp. t-conorm 2 ) is called t-representable if there exists a fuzzy t-norm $T$ and a fuzzy t-conorm $S$ (resp. a fuzzy t-conorm $S$ and fuzzy t-norm $T$ ) such that $\forall a=\left(a_{1}, a_{2}\right), b=\left(b_{1}, b_{2}\right) \in L^{*}, \tau(a, b)=\left(T\left(a_{1}, b_{1}\right), S\left(a_{2}, b_{2}\right)\right)$ (resp. $\partial(a, b))=\left(S\left(a_{1}, b_{1}\right), T\left(a_{2}, b_{2}\right)\right)$.

$T$ and $S$ (resp. $S$ and $T$ ) are called the representants of $\tau$ (resp. 2).

The theorem below states conditions under which a pair of connectives on $[0,1]$ gives rise to a t-representable intuitionistic fuzzy t-norm ( $\mathrm{t}$-conorm).

Theorem 2.4 (see Cornelis et al. [20, Theorem 2, pages 60-61]). Given a fuzzy t-norm $T$ and $a$ fuzzy t-conorm $S$ satisfying $\forall a_{1}, a_{2} \in[0,1], T\left(a_{1}, a_{2}\right) \leq 1-S\left(1-a_{1}, 1-a_{2}\right)$.

The mappings $\tau$ and $\partial$ defined by, for $x=\left(x_{1}, x_{2}\right)$ and $y=\left(y_{1}, y_{2}\right)$ in $L^{*}: \tau(x, y)=$ $\left(T\left(x_{1}, y_{1}\right), S\left(x_{2}, y_{2}\right)\right)$ and $\partial(x, y)=\left(S\left(x_{1}, y_{1}\right), T\left(x_{2}, y_{2}\right)\right)$, are, respectively, a t-representable intuitionistic fuzzy t-norm and $t$-representable intuitionistic fuzzy t-conorm.

Throughout the paper, we consider only continuous t-representable intuitionistic fuzzy t-conorms (shortly if-t-conorm) and continuous t-representable intuitionistic fuzzy tnorms (shortly if-t-norm). They are denoted by $\mathcal{\partial}=(S, T)$ and $\tau=(T, S)$, respectively, where $\forall a, b \in[0,1], S(a, b) \leq 1-T(1-a, a-b)$.

From the previous result, we deduce some examples of if-t-norms and if-t-conorms.

Example 2.5. (1) $\tau_{\mathbf{M}}=\left(T_{\mathbf{M}}, S_{\mathbf{M}}\right)$ and $\partial_{\mathbf{M}}=\left(S_{\mathbf{M}}, T_{\mathbf{M}}\right)$ are, respectively, if-t-norm and if-tconorm associated to $T_{\mathbf{M}}$ and $S_{\mathbf{M}}$ since $\forall a, b \in[0,1], T_{\mathbf{M}}(a, b) \leq 1-S_{\mathbf{M}}(1-a, 1-b)$.

(2) $\tau_{\mathrm{L}}=\left(T_{\mathrm{L}}, S_{\mathrm{L}}\right)$ and $\partial_{\mathrm{L}}=\left(S_{\mathrm{L}}, T_{\mathrm{L}}\right)$ are, respectively, if-t-norm and if-t-conorm associated to $T_{\mathrm{L}}$ and $S_{\mathrm{L}}$ since $\forall a, b \in[0,1], T_{\mathrm{L}}(a, b) \leq 1-S_{\mathrm{L}}(1-a, 1-b)$.

(3) $\tau_{\mathbf{P}}=\left(T_{\mathbf{P}}, S_{\mathbf{P}}\right)$ and $\partial_{\mathbf{P}}=\left(S_{\mathbf{P}}, T_{\mathbf{P}}\right)$ are, respectively, if-t-norm and if-t-conorm associated to $T_{\mathbf{P}}$ and $S_{\mathbf{P}}$ since $\forall a, b \in[0,1], T_{\mathbf{P}}(a, b) \leq 1-S_{\mathbf{P}}(1-a, 1-b)$.

Definition 2.6 (see Cornelis et al. [20, Definition 8, page 64]). (1) The intuitionistic fuzzy Rimplication (shortly if-R-implication) associated with an if-t-norm $\tau=(T, S)$ is a binary operation on $L^{*}$ defined by: $\forall x=\left(x_{1}, x_{2}\right), y=\left(y_{1}, y_{2}\right) \in L^{*}, I_{\tau}(x, y)=\sup \{z \in$ $\left.L^{*}, \tau(x, z) \leq_{L^{*}} y\right\}=\sup \left\{z=\left(z_{1}, z_{2}\right) \in L^{*}, T\left(x_{1}, z_{1}\right) \leq y_{1}\right.$ and $\left.S\left(x_{2}, z_{2}\right) \geq y_{2}\right\}$.

(2) The intuitionistic fuzzy coimplicator (shortly if-coimplicator) associated with an if-t-conorm $\partial=(S, T)$ is a binary operation on $L^{*}$ defined by: $\forall x=\left(x_{1}, x_{2}\right), y=$ $\left(y_{1}, y_{2}\right) \in L^{*}, J_{2}(x, y)=\inf \left\{z \in L^{*}, y L_{L^{*}} \partial(x, z)\right\}=\inf \left\{z=\left(z_{1}, z_{2}\right) \in L^{*} / y_{1} \leq\right.$ $S\left(x_{1}, z_{1}\right)$ and $\left.y_{2} \geq T\left(x_{2}, z_{2}\right)\right\}$.

We establish in the sequel some new and basic results on the previous implications. These results will be useful later. 


\subsection{Some Basic Results on Fuzzy Implications and If-Implications}

The following result establishes two links between the fuzzy R-implication $I_{T}$ and the fuzzy coimplicator $J_{S}$.

Proposition 2.7. Let $S$ and $T$ such that $\forall a, b \in[0,1], T(a, b) \leq 1-S(1-a, 1-b)$. Then

(1) for all $a, b \in[0,1], I_{T}(a, b) \geq 1-J_{S}(1-a, 1-b)$;

(2) if $T$ and $S$ are dual, then $\forall a, b \in[0,1], I_{T}(a, b)=1-J_{S}(1-a, 1-b)$.

The following result gives expressions of an if-R-implication and an if-coimplicator by means of $I_{T}$ and $J_{S}$.

Lemma 2.8. For all $x=\left(x_{1}, x_{2}\right), y=\left(y_{1}, y_{2}\right) \in L^{*}$,

(1) $J_{2}(x, y)=\left(J_{S}\left(x_{1}, y_{1}\right), \min \left(I_{T}\left(x_{2}, y_{2}\right), 1-J_{S}\left(x_{1}, y_{1}\right)\right)\right)$;

(2) $I_{\tau}(x, y)=\left(\min \left(I_{T}\left(x_{1}, y_{1}\right), 1-J_{S}\left(x_{2}, y_{2}\right)\right), J_{S}\left(x_{2}, y_{2}\right)\right)$.

We now introduce a new condition which can be satisfied by a if-t-conorm $\partial=(S, T)$.

Definition 2.9. $2=(S, T)$ satisfies condition $G$ if

$$
\forall\left(a_{1}, a_{2}\right),\left(b_{1}, b_{2}\right) \in L^{*}, \quad\left\{\begin{array}{l}
a_{1}>b_{1} \\
a_{2}<b_{2} \\
a_{1}+a_{2}=b_{1}+b_{2}
\end{array} \Longrightarrow I_{T}\left(b_{2}, a_{2}\right)+J_{S}\left(b_{1}, a_{1}\right) \leq 1 .\right.
$$

Let us end this section by giving some examples of if-t-conorms satisfying condition $G$. This justifies that the class of continuous t-representable if-t-conorms satisfying condition $G$ is not empty.

Proposition 2.10. (1) For all $l \in[0,+\infty], \partial_{l}^{F}=\left(S_{l}^{F}, T_{l}^{F}\right)$ satisfies condition $G$.

(2) If $S$ and $T$ are dual, then the restriction of $\partial=(S, T)$ on $L_{1}^{*}=\left\{\left(x_{1}, x_{2}\right) \in L^{*}, x_{1}+x_{2}=1\right\}$ satisfies condition $G$.

In the next section, we recall some basic notions on IFRs and study its standard completeness (see Atanassov [12], Bustince and Burillo [15], and Dimitrov [17, 18]). We establish, for a given $\tau=(T, S)$, a characterization of the $\tau$-transitivity of an IFR $R$.

\section{Preliminaries on IFRs}

\subsection{Review on IFRs}

An IFS in $X$ is an expression $A$ given by $A=\left\{\left\langle x, \mu_{A}(x), v_{A}(x)\right\rangle, x \in X\right\}$, where $\mu_{A}: X \rightarrow$ $[0,1]$ and $v_{A}: X \rightarrow[0,1]$ are functions satisfying the condition $\forall x \in X, \mu_{A}(x)+v_{A}(x) \leq 1$. The numbers $\mu_{A}(x)$ and $v_{A}(x)$ denote, respectively, the degree of membership and the degree of nonmembership of the element $x$ in $A$. The number $\pi_{A}(x)=1-\mu_{A}(x)-v_{A}(x)$ is an index 
of the element $x$ in $X$. Obviously, when $\forall x \in X, v_{A}(x)=1-\mu_{A}(x)$, that is, $\pi_{A}(x)=0$, the IFS $A$ is a fuzzy set (simply denoted by FS) in $X$. In this case, $\forall x \in X, A(x)=\mu_{A}(x)$.

Let $A$ and $B$ be two IFSs, and let $\partial=(S, T)$. The intuitionistic fuzzy union $A \cup_{2} B$ associated to 2 is an IFS defined by

$$
\forall x \in X, \quad\left\{\begin{array}{l}
\mu_{A \cup_{2} B}(x)=S\left(\mu_{A}(x), \mu_{B}(x)\right), \\
v_{A \cup_{2} B}(x)=T\left(v_{A}(x), v_{B}(x)\right)
\end{array}\right.
$$

(we recall that if $A$ and $B$ are FSs, and $T$ and $S$ are dual, then $A \cup_{2} B$ becomes the well-known fuzzy union $A \cup_{S} B$ defined by $\forall x \in X, A \cup_{S} B(x)=S(A(x), B(x))$. And if $A$ and $B$ are crisp, $A \cup_{2} B=A \cup_{S} B$ becomes the crisp union). As defined in the Introduction, an IFR in $X$ is an IFS in $X \times X$.

We complete some basic definitions on IFRs.

Definition 3.1. Let $R$ be an IFR.

(1) $R$ is reflexive if $\forall x \in X, \mu_{R}(x, x)=1$.

(2) $R$ is symmetric if $\forall x, y \in X, \mu_{R}(x, y)=\mu_{R}(y, x)$ and $v_{R}(x, y)=v_{R}(y, x)$.

(3) $R$ is $\pi$-symmetric if $\forall x, y \in X, \pi_{R}(x, y)=\pi_{R}(y, x)$.

(4) $R$ is perfect antisymmetric if $\forall(x, y) \in X \times X, x \neq y$,

$$
\left(\begin{array}{c}
\mu_{R}(x, y)>0 \\
\text { or } \\
\left(\mu_{R}(x, y)=0, v_{R}(x, y)<1\right)
\end{array}\right) \Longrightarrow\left\{\begin{array}{l}
\mu_{R}(y, x)=0 \\
v_{R}(y, x)=1
\end{array}\right.
$$

(5) The converse of $R$ is the IFR denoted $R^{-1}$ and defined by $\forall x, y \in X, \mu_{R^{-1}}(x, y)=$ $\mu_{R}(y, x)$ and $v_{R^{-1}}(x, y)=v_{R}(y, x)$.

In the following, we recall the well-known notion of completeness of a crisp relation in X. We then present definition of the standard completeness of a FR and its two usual and particular cases (weak completeness and strong completeness). Following that line, we introduce the definition of the standard completeness of an IFR. We establish a link between that standard definition and the one introduced by Dimitrov (see $[17,18])$. And we write the two particular cases of that standard definition.

\subsection{Intuitionistic Fuzzy Standard Completeness (2-Completeness)}

Let $R$ be a reflexive IFR and $\partial=(S, T)$.

When $R$ is a crisp relation, $R$ is complete if $R \cup R^{-1}=X^{2}$, that is, $\forall x, y \in X, x R y$ or $y R x$.

When $R$ is a FR, for the fuzzy t-conorm $S, R$ is $S$-complete if $R \cup_{S} R^{-1}=X^{2}$, that is, $\forall x, y \in X, S(R(x, y), R(y, x))=1$. In particular, if $S=S_{M}$, we simply say that $R$ is strongly complete, that is, $\forall x, y \in X, \max (R(x, y), R(y, x))=1$. If $S=S_{L}$, we simply say that $R$ is weakly complete, that is, $\forall x, y \in X, R(x, y)+R(y, x) \geq 1$ (see Fono and Andjiga [7, Definition 2, page 375]). 
In the general case where $R$ is an IFR and $\partial=(S, T)$, we have the following generic version of the standard completeness of $R$.

Definition 3.2. $R$ is 2-complete if $R \cup_{2} R^{-1}=X^{2}$, that is,

$$
\forall x, y \in X, \quad\left\{\begin{array}{l}
S\left(\mu_{R}(x, y), \mu_{R}(y, x)\right)=1 \\
T\left(v_{R}(x, y), v_{R}(y, x)\right)=0
\end{array}\right.
$$

Remark 3.3. If an IFR $R$ becomes a FR, and $S$ and $T$ are dual, then $(S, T)$-completeness becomes $S$-completeness. Furthermore, if $R$ becomes crisp, then 2 -completeness and $S$-completeness become crisp completeness.

Dimitrov (see [17, Definition 2, page 151]) introduced the following version of completeness of an IFR: $R$ is $D$-complete if $\forall x, y \in X$,

$$
\left[\left(\mu_{R}(x, y), v_{R}(x, y)\right)=(0,1)\right] \Longrightarrow\left[\mu_{R}(y, x)>0, v_{R}(y, x)<1\right]
$$

It is important to notice that $D$-completeness is not a version of the standard completeness. However, the following result shows that it is weaker than each version of the standard completeness.

Proposition 3.4. If $R$ is $(S, T)$-complete, then $R$ is $D$-complete.

As for FRs, we deduce the two following interesting particular cases of 2 completeness when $\partial \in\left\{\partial_{\mathrm{M}}, \partial_{\mathrm{L}}\right\}$.

Example 3.5. Let $R$ be a reflexive IFR and $\partial=(S, T)$.

(1) If $\partial=\partial \mathrm{M}=(\max , \min )$, then $R$ is $\partial$-complete if $\forall x, y \in X$,

$$
\begin{aligned}
& \max \left(\mu_{R}(x, y), \mu_{R}(y, x)\right)=1, \\
& \min \left(v_{R}(x, y), v_{R}(y, x)\right)=0 .
\end{aligned}
$$

In this case, we simply say that $R$ is strongly complete.

(2) If $\partial=\partial_{\mathrm{L}}=\left(S_{\mathrm{L}}, T_{\mathrm{L}}\right)$, then $R$ is $\partial$-complete if $\forall x, y \in X$,

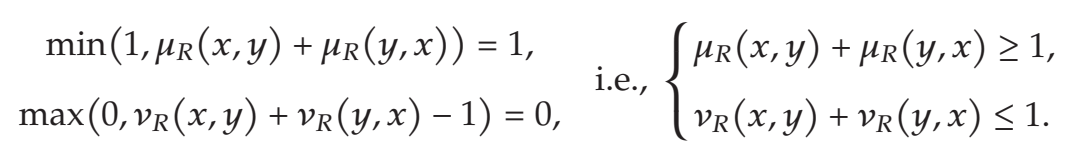

In this case, we simply say that $R$ is weakly complete.

We notice that, if $R$ becomes a FR, then intuitionistic strong completeness of $R$ and the intuitionistic weak completeness of $R$ become, respectively, fuzzy strong completeness of $R$ and fuzzy weak completeness of $R$. Furthermore, as for FRs, intuitionistic strong completeness implies intuitionistic weak completeness. 
Throughout the paper, $R$ is a reflexive, weakly complete, and $\pi$-symmetric IFR.

In the sequel, we define $\tau$-transitivity of an IFR $R$. We introduce and analyze four elements of $L^{*}$. They enable us to obtain a characterization of the $\tau$-transitivity of $R$ which generalizes the one obtained earlier by Fono and Andjiga [7] for FRs.

\section{3. 乙-transitivity of an IFR: Definition and Characterization}

Let $\tau=(T, S)$ and $I \tau$ be the if-R-implication associated to $\tau$.

Definition 3.6. $R$ is $\tau$-transitive if $\forall x, y, z \in X$,

$$
\tau(R(x, y), R(y, z)) \leq_{L^{*}} R(x, z), \quad \text { i.e., }\left\{\begin{array}{l}
\mu_{R}(x, z) \geq T\left(\mu_{R}(x, y), \mu_{R}(y, z)\right) \\
v_{R}(x, z) \leq S\left(v_{R}(x, y), v_{R}(y, z)\right)
\end{array}\right.
$$

If $R$ becomes a FR and $T$ and $S$ are dual, then the $\tau$-transitivity becomes the usual $T$-transitivity, that is, $\forall x, y, z \in X, R(x, z) \geq T(R(x, y), R(y, z))$. If $R$ becomes a crisp relation, then the $\tau$-transitivity and the $T$-transitivity become the crisp transitivity, that is, $\forall x, y, z \in$ $X,(x R y$ and $y R z) \Rightarrow x R z$.

We write the particular case of the $\tau$-transitivity where $\tau=\tau_{\mathbf{M}}$.

Example 3.7. If $\tau=\tau_{\mathrm{M}}$, then $\mathrm{R}$ is $\tau_{\mathrm{M}}$-transitive if $\forall x, y, z \in X$,

$$
\begin{aligned}
& \mu_{R}(x, z) \geq \min \left(\mu_{R}(x, y), \mu_{R}(y, z)\right), \\
& v_{R}(x, z) \leq \max \left(v_{R}(x, y), v_{R}(y, z)\right) .
\end{aligned}
$$

We simply say that $R$ is transitive.

To establish a characterization of the $\tau$-transitivity of $R$, we need the following four elements of $[0,1]^{2}$ associated to $R$.

Let us introduce and analyze these elements of $[0,1]^{2}$.

Definition 3.8. For all $x, y, z \in X$,

(1) $\left(\alpha_{1}(x, y, z), \beta_{1}(x, y, z)\right)=\left(T\left(\mu_{R}(z, y), \mu_{R}(y, x)\right), S\left(v_{R}(z, y), v_{R}(y, x)\right)\right)$;

(2) $\left(\alpha_{2}(x, y, z), \beta_{2}(x, y, z)\right)=\left(T\left(\mu_{R}(x, y), \mu_{R}(y, z)\right), S\left(v_{R}(x, y), v_{R}(y, z)\right)\right)$;

(3) $\left(\alpha_{3}(x, y, z), \beta_{3}(x, y, z)\right)=\min \left(I_{\tau}\left[\left(\mu_{R}(y, z), v_{R}(y, z)\right),\left(\mu_{R}(y, x), v_{R}(y, x)\right)\right], I_{\tau}\left[\left(\mu_{R}(x\right.\right.\right.$, $\left.\left.\left.y), v_{R}(x, y)\right),\left(\mu_{R}(z, y), v_{R}(z, y)\right)\right]\right)$;

(4) $\left(\alpha_{4}(x, y, z), \beta_{4}(x, y, z)\right)=\min \left(I_{\tau}\left[\left(\mu_{R}(y, x), v_{R}(y, x)\right),\left(\mu_{R}(y, z), v_{R}(y, z)\right)\right], I_{\tau}\left[\left(\mu_{R}(z\right.\right.\right.$, $\left.\left.\left.y), v_{R}(z, y)\right),\left(\mu_{R}(x, y), v_{R}(x, y)\right)\right]\right)$.

The next result shows that $\left(\alpha_{i}(x, y, z), \beta_{i}(x, y, z)\right)_{i \in\{1,2,3,4\}}$ are elements of $L^{*}$ and deduces expressions of $\alpha_{3}(x, y, z), \beta_{3}(x, y, z), \alpha_{4}(x, y, z)$ and $\beta_{4}(x, y, z)$. 
Proposition 3.9. (1) For all $i \in\{1,2,3,4\},\left(\alpha_{i}(x, y, z), \beta_{i}(x, y, z)\right) \in L^{*}$.

(2) (i) $\alpha_{3}(x, y, z)$ is the minimum of $\min \left[I_{T}\left(\mu_{R}(x, y), \mu_{R}(z, y)\right), 1-J_{S}\left(v_{R}(x, y)\right.\right.$, $\left.\left.v_{R}(z, y)\right)\right]$ and $\min \left[I_{T}\left(\mu_{R}(y, z), \mu_{R}(y, x)\right), 1-J_{S}\left(v_{R}(y, z), v_{R}(y, x)\right)\right]$.

(ii) $\beta_{3}(x, y, z)=\max \left(J_{S}\left(v_{R}(y, z), v_{R}(y, x)\right), J_{S}\left(v_{R}(x, y), v_{R}(z, y)\right)\right)$.

(iii) $\alpha_{4}(x, y, z)$ is the minimum of $\min \left[I_{T}\left(\mu_{R}(z, y), \mu_{R}(x, y)\right), 1-J_{S}\left(v_{R}(z, y)\right.\right.$, $\left.\left.v_{R}(x, y)\right)\right]$ and $\left.\min \left[I_{T}\left(\mu_{R}(y, x), \mu_{R}(y, z)\right), 1-J_{S}\left(v_{R}(y, x), v_{R}(y, z)\right)\right)\right]$.

(iv) $\beta_{4}(x, y, z)=\max \left(J_{S}\left(v_{R}(y, x), v_{R}(y, z)\right), J_{S}\left(v_{R}(z, y), v_{R}(x, y)\right)\right)$.

The following remark gives some comparisons of those elements of $L^{*}$.

Remark 3.10. For all $x, y, z \in X$,

(1) $\left(\alpha_{1}(x, y, z), \beta_{1}(x, y, z)\right) \leq_{L^{*}}\left(\alpha_{3}(x, y, z), \beta_{3}(x, y, z)\right)$;

(2) $\left(\alpha_{2}(x, y, z), \beta_{2}(x, y, z)\right) \leq_{L^{*}}\left(\alpha_{4}(x, y, z), \beta_{4}(x, y, z)\right)$.

The following result shows that in the particular case where $R$ is strongly complete, the four reals $\alpha_{3}(x, y, z), \alpha_{4}(x, y, z), \beta_{3}(x, y, z)$ and $\beta_{4}(x, y, z)$ become simple.

Corollary 3.11. Let $R$ be an IFR, and let $x, y, z \in X$.

(1) If $R$ is strongly complete and

$$
\left(\left\{\begin{array} { l } 
{ \mu _ { R } ( y , x ) < \mu _ { R } ( x , y ) } \\
{ \mu _ { R } ( z , y ) < \mu _ { R } ( y , z ) }
\end{array} \text { or } \left\{\begin{array}{l}
\mu_{R}(y, x)=\mu_{R}(x, y) \\
\mu_{R}(z, y)<\mu_{R}(y, z)
\end{array} \text { or }\left\{\begin{array}{l}
\mu_{R}(y, x)<\mu_{R}(x, y) \\
\mu_{R}(z, y)=\mu_{R}(y, z)
\end{array}\right)\right.\right.\right. \text {, }
$$

then

$$
\begin{gathered}
\alpha_{1}(x, y, z)=T\left(\mu_{R}(z, y), \mu_{R}(y, x)\right)<1 \\
\alpha_{3}(x, y, z)=\min \left(\mu_{R}(y, x), \mu_{R}(z, y)\right) \\
\alpha_{2}(x, y, z)=\alpha_{4}(x, y, z)=1 .
\end{gathered}
$$

(2) If $R$ is strongly complete and

$$
\left(\left\{\begin{array} { l } 
{ v _ { R } ( y , x ) > v _ { R } ( x , y ) } \\
{ v _ { R } ( z , y ) > v _ { R } ( y , z ) }
\end{array} \text { or } \left\{\begin{array}{l}
v_{R}(y, x)=v_{R}(x, y) \\
v_{R}(z, y)>v_{R}(y, z)
\end{array} \text { or }\left\{\begin{array}{l}
v_{R}(y, x)>v_{R}(x, y) \\
v_{R}(z, y)=v_{R}(y, z)
\end{array}\right),\right.\right.\right.
$$

then

$$
\begin{gathered}
\beta_{1}(x, y, z)=S\left(v_{R}(z, y), v_{R}(y, x)\right)>0, \\
\beta_{3}(x, y, z)=\max \left(v_{R}(y, x), v_{R}(z, y)\right), \\
\beta_{2}(x, y, z)=\beta_{4}(x, y, z)=0 .
\end{gathered}
$$


In the particular case where $T$ and $S$ are dual and $R$ becomes a FR, we have some links between $\alpha_{i}$ and $\beta_{i}$ for $i \in\{1,2,3,4\}$. Furthermore, we obtain expressions of $\left(\alpha_{i}(x, y, z)\right)_{i \in\{1,2,3,4\}}$ introduced earlier by Fono and Andjiga (see [7, page 375]).

Corollary 3.12. If $T$ and $S$ are dual and $R$ is a FR, then $\forall x, y, z \in X$,

(1)

$$
\begin{aligned}
& \beta_{1}(x, y, z)=1-\alpha_{1}(x, y, z), \\
& \beta_{2}(x, y, z)=1-\alpha_{2}(x, y, z), \\
& \beta_{3}(x, y, z)=1-\alpha_{3}(x, y, z), \\
& \beta_{4}(x, y, z)=1-\alpha_{4}(x, y, z) .
\end{aligned}
$$

(2)

$$
\begin{gathered}
\alpha_{1}(x, y, z)=T\left(\mu_{R}(z, y), \mu_{R}(y, x)\right), \\
\alpha_{2}(x, y, z)=T\left(\mu_{R}(x, y), \mu_{R}(y, z)\right), \\
\alpha_{3}(x, y, z)=\min \left(I_{T}\left(\mu_{R}(x, y), \mu_{R}(z, y)\right), I_{T}\left(\mu_{R}(y, z), \mu_{R}(y, x)\right)\right), \\
\alpha_{4}(x, y, z)=\min \left(I_{T}\left(\mu_{R}(y, x), \mu_{R}(y, z)\right), I_{T}\left(\mu_{R}(z, y), \mu_{R}(x, y)\right)\right) .
\end{gathered}
$$

We end this section by establishing by means of those four elements of $L^{*}$ a characterization of the $\tau$-transitivity of an IFR $R$. Before that, let us recall a characterization of the $T$-transitivity of a FR: $\forall x, y, z \in X$, if $T$ and $S$ are dual, and the IFR $R$ becomes a FR, then Fono and Andjiga (see [7, Lemma 1, page 375]) used the four reals $\alpha_{1}(x, y, z), \alpha_{2}(x, y, z), \alpha_{3}(x, y, z)$, and $\alpha_{4}(x, y, z)$ defined in Corollary 3.12, to obtain the following characterization of the $T$-transitivity of $R$.

$\mathrm{R}$ is $T$-transitive on $\{x, y, z\}$ if and only if

$$
\begin{aligned}
& R(x, z)=\mu_{R}(x, z) \in\left[\alpha_{2}(x, y, z), \alpha_{4}(x, y, z)\right], \\
& R(z, x)=\mu_{R}(z, x) \in\left[\alpha_{1}(x, y, z), \alpha_{3}(x, y, z)\right] .
\end{aligned}
$$

We generalize that result for an IFR. Therefore, we obtain our first key result.

Lemma 3.13. Let $\{x, y, z\} \subseteq X$.

The two following statements are equivalent:

(i) $R$ is $\tau$-transitive on $\{x, y, z\}$;

(ii)

$$
\begin{array}{ll}
\mu_{R}(x, z) \in\left[\alpha_{2}(x, y, z), \alpha_{4}(x, y, z)\right], & v_{R}(x, z) \in\left[\beta_{4}(x, y, z), \beta_{2}(x, y, z)\right], \\
\mu_{R}(z, x) \in\left[\alpha_{1}(x, y, z), \alpha_{3}(x, y, z)\right], & v_{R}(z, x) \in\left[\beta_{3}(x, y, z), \beta_{1}(x, y, z)\right] .
\end{array}
$$


In the following section, we study a factorization of $R$. For that, we proceed as follows: (i) we recall the factorization of an IFR established by Dimitrov [18]; (ii) we notice some similarities between that factorization and those established earlier on FRs by Dutta [3], Richardson [10], and Fono and Andjiga [7]; (iii) using the vocabulary used by these authors for the factorization of FRs, we write Dimitrov's [18] factorization in a simple and elegant way for the (max, $\min$ ) if-t-conorm (see Lemma 4.2); (iv) we point out some intuitive difficulties of the strict component obtained in [18]; (v) we introduce definitions of an indifference and a strict component of an IFR, and we complete Lemma 4.2 to obtain a general factorization of an IFR when the union is defined by means of a given continuous t-representable if-t-conorm satisfying condition $G$.

\section{Factorization of an IFR}

\subsection{Review on Dimitrov's Results and Some Comments}

Dimitrov proposed a factorization of an IFR and obtained the following result.

Proposition 4.1 (see Dimitrov [18, Proposition 1], or Dimitrov [17, Proposition 3, page 152]). Let $\partial=\partial_{M}=(\max , \min )$ be the if-t-conorm, and let $R$ be an IFR which is reflexive, $D$-complete and $\pi$-symmetric; I and $P$ are two IFRs such that

(i) $R=I \cup_{2 M} P$,

(ii) $I$ is symmetric,

(iii) $P$ is perfect antisymmetric,

(iv)

$$
\forall(x, y) \in X \times X, \quad\left\{\begin{array} { l } 
{ \mu _ { R } ( x , y ) = \mu _ { R } ( y , x ) } \\
{ v _ { R } ( x , y ) = v _ { R } ( y , x ) }
\end{array} \Longrightarrow \left\{\begin{array}{l}
\mu_{P}(x, y)=\mu_{P}(y, x) \\
v_{P}(x, y)=v_{P}(y, x)
\end{array}\right.\right.
$$

Then, for all $x, y \in X$,

(1) $I(x, y)=\left(\mu_{I}(x, y), v_{I}(x, y)\right)$, where

$$
\begin{aligned}
& \mu_{I}(x, y)=\min \left(\mu_{R}(x, y), \mu_{R}(y, x)\right), \\
& v_{I}(x, y)=\max \left(v_{R}(x, y), v_{R}(y, x)\right) ;
\end{aligned}
$$

(2) $P(x, y)=\left(\mu_{P}(x, y), v_{P}(x, y)\right)$, where

$$
\begin{aligned}
& \mu_{P}(x, y)= \begin{cases}\mu_{R}(x, y), & \text { if } \mu_{R}(x, y)>\mu_{R}(y, x), \\
0, & \text { otherwise, }\end{cases} \\
& v_{P}(x, y)= \begin{cases}v_{R}(x, y), & \text { if } v_{R}(x, y)>v_{R}(y, x), \\
1, & \text { otherwise. }\end{cases}
\end{aligned}
$$


After a careful check, we notice that, in the particular case where $R$ becomes a FR, conditions (4.1) and (4.3) become some known notions introduced earlier by Dutta [3] and, used by Richardson [10] and, Fono and Andjiga [7].

Let $R$ be an IFR.

(1) If $R$ becomes a FR, the strict component $P$ of $R$ becomes the fuzzy strict component of $R$ and thus, condition (4.1) of the previous result becomes condition " $P$ is simple," that is, $\forall(x, y) \in X \times X$,

$$
\begin{aligned}
& R(x, y)=\mu_{R}(x, y)=R(y, x)=\mu_{R}(y, x) \\
& \Longrightarrow P(x, y)=\mu_{P}(x, y)=P(y, x)=\mu_{P}(y, x) .
\end{aligned}
$$

For convenience and as in fuzzy case, we also call condition (4.1): "P is simple."

(2) The strict component $P$ of $R$ obtained in the previous result satisfies the following condition:

$$
\forall x, y \in X, \quad\left\{\begin{array} { l } 
{ \mu _ { R } ( x , y ) \leq \mu _ { R } ( y , x ) } \\
{ v _ { R } ( x , y ) \geq v _ { R } ( y , x ) }
\end{array} \Longleftrightarrow \left\{\begin{array}{l}
\mu_{P}(x, y)=0 \\
v_{P}(x, y)=1
\end{array}\right.\right.
$$

If $R$ becomes a FR, condition (4.5) becomes the condition " $P$ is regular," that is, $\forall(x, y) \in X \times X$,

$$
R(x, y)=\mu_{R}(x, y) \leq R(y, x)=\mu_{R}(y, x) \Longrightarrow P(x, y)=\mu_{P}(x, y)=0 .
$$

For convenience and as in fuzzy case, we also call condition (4.5): " $P$ is regular."

With these remarks on the intuitionistic fuzzy strict component obtained in Dimitrov [18], we rewrite Proposition 4.1 as follows:

" $P$ is regular and $I$ is defined by (4.2) if $P$ is perfect antisymmetric and simple, $I$ is symmetric, and $R=I \cup_{2} P$ for $\partial=\partial_{M}=(\max , \min ) . "$

An interesting question is to check if this version of Dimitrov's result remains true for $\partial=(S, T)$.

The following result shows that this is true. More precisely, it establishes a generalization of the previous version of Dimitrov's result. And we obtain our second key result.

Lemma 4.2. Let $\partial=(S, T), R$ be a reflexive, weakly complete and $\pi$-symmetric IFR; $I$ and $P$ are two IFRs such that: (i) $R=I \cup_{2} P$, (ii) $I$ is symmetric, (iii) $P$ is perfect antisymmetric, (iv) $P$ is simple. Then,

(1) I is defined by (4.2);

(2) $P$ is regular.

Otherwise, let us also point out some intuitive difficulties of the strict component obtained by Dimitrov in the factorization of Proposition 4.1. 
(1) The component $P$ defined by (4.3) is obtained for the particular t-representable if$\mathrm{t}$-conorm $\partial=\partial_{\mathrm{M}}=(\max , \min )$.

(2) The discontinuity of $P$, that is,

(i) for all $x, y \in X$, the degree $\mu_{P}(x, y)$ is insensitive for the variability of $\mu_{R}(x, y)$ and $\mu_{R}(y, x)$. For illustration, if $\left(\mu_{R}(x, y), \mu_{R}(y, x)\right)=(1,0.999)$ or $\left(\mu_{R}(x, y), \mu_{R}(y, x)\right)=(1,0)$, then $\mu_{P}(x, y)=1$. But if $\left(\mu_{R}(x, y), \mu_{R}(y, x)\right)=$ $(1,1)$, then $\mu_{P}(x, y)=0$;

(ii) for all $x, y \in X$, the degree $v_{P}(x, y)$ is insensitive for the variability of $v_{R}(x, y)$ and $v_{R}(y, x)$. For illustration, if $\left(v_{R}(x, y), v_{R}(y, x)\right)=(0,0.001)$ or $\left(v_{R}(x, y), v_{R}(y, x)\right)=(0,1)$, then $v_{P}(x, y)=0$. But if $\left(v_{R}(x, y), v_{R}(y, x)\right)=(0,0)$, then $v_{P}(x, y)=1$.

The previous observations force us to complete and generalize the factorization of Dimitrov for a if-t-conorm $\mathcal{\partial}=(S, T)$ satisfying condition $G$.

\subsection{A New and General Factorization of an IFR}

First at all, we introduce formally a definition of "indifference of an IFR" and "strict component of an IFR."

Definition 4.3. Let $\partial=(S, T)$ satisfying condition $G, R$ be an IFR; $I$ and $P$ are two IFRs. $I$ and $P$ are "indifference of $R$ " and "strict component of $R$ " associated to 2 , respectively, if the following conditions are satisfied:

$$
R=I \cup_{(S, T)} P,
$$

$P$ is simple and perfect antisymmetric,

$I$ is symmetric.

With the results of Lemma 4.2, the equality of (4.7) becomes the following equation:

$$
\forall x, y \in X, \quad\left(\mu_{R}(x, y), v_{R}(x, y)\right)=2\left[\left(\mu_{R}(y, x), v_{R}(y, x)\right),(a, b)\right]
$$

which is equivalent to the following system:

$$
\begin{gathered}
a+b \leq 1, \\
S\left(\mu_{R}(y, x), a\right)=\mu_{R}(x, y) \quad\left(E_{1}\right), \\
T\left(v_{R}(y, x), b\right)=v_{R}(x, y) \quad\left(E_{2}\right) .
\end{gathered}
$$

To establish a new and general factorization, we need the following lemma which is our third key result. 
Lemma 4.4. Let $\partial=(S, T), R$ be an IFR, and let $x, y \in X$ such that

$$
\begin{aligned}
& \mu_{R}(x, y)>\mu_{R}(y, x), \\
& v_{R}(x, y)<v_{R}(y, x) .
\end{aligned}
$$

Then,

(i) $(4.9)\left(E_{1}\right)$ and $(4.9)\left(E_{2}\right)$ have at least one solution;

(ii) each solution of $(4.9)\left(E_{1}\right)$ is strictly positive, and each solution of $(4.9)\left(E_{2}\right)$ is strictly least than 1;

(iii) if 2 satisfies condition $G$, then (4.8) or (4.9) has at least one solution;

(iv) if 2 satisfies condition $G$, then the element $\left(J_{S}\left(\mu_{R}(y, x), \mu_{R}(x, y)\right), I_{T}\left(v_{R}(y, x), v_{R}(x, y)\right)\right)$ is the optimal solution of (4.8) or (4.9), that is, $I_{T}\left(v_{R}(y, x), v_{R}(x, y)\right)$ is the upper solution of $(4.9)\left(E_{2}\right)$, and $J_{S}\left(\mu_{R}(y, x), \mu_{R}(x, y)\right)$ is the lowest solution of $(4.9)\left(E_{1}\right)$.

(v) furthermore, if $\partial=\partial_{M}=(\max , \min )$ or $\partial$ is a strict if-t-conorm (i.e., $S$ and $T$ are strict) satisfying condition $G$, then $\left(J_{S}\left(\mu_{R}(y, x), \mu_{R}(x, y)\right), I_{T}\left(v_{R}(y, x), v_{R}(x, y)\right)\right)$ is the unique solution of (4.8) or (4.9).

We now establish the result of factorization which is the first main result of our paper.

Theorem 4.5. Let $\partial=(S, T)$ satisfying condition $G, R$ be an IFR; I and $P$ are two IFRs.

The two following statements are equivalent:

(1) I and P are "indifference" and "strict component of $R$ " associated to 2, respectively;

(2) (i)

$$
\forall x, y \in X, \quad\left\{\begin{array}{l}
\mu_{I}(x, y)=\mu_{I}(y, x)=\min \left(\mu_{R}(x, y), \mu_{R}(y, x)\right) \\
v_{I}(x, y)=v_{I}(y, x)=\max \left(v_{R}(x, y), v_{R}(y, x)\right)
\end{array}\right.
$$

(ii) $\forall x, y \in X, \exists\left(c_{x y}, g_{x y}\right) \in L^{*}$ such that $c_{x y}>0, c_{x y}$ is a solution of $(4.9)\left(E_{1}\right), g_{x y}<$ $1, g_{x y}$ is a solution of $(4.9)\left(E_{2}\right)$, and

$$
\begin{aligned}
& \mu_{P}(x, y)= \begin{cases}0, & \text { if } \mu_{R}(x, y) \leq \mu_{R}(y, x), \\
c_{x y}, & \text { otherwise, }\end{cases} \\
& v_{P}(x, y)= \begin{cases}1, & \text { if } v_{R}(x, y) \geq v_{R}(y, x), \\
g_{x y}, & \text { otherwise. }\end{cases}
\end{aligned}
$$

The previous factorization gives a unique indifference of $R$. However, as in fuzzy case and contrary to the crisp case, for an IFR $R$ and for $\partial=(S, T)$ satisfying $G$, the previous result generates a family of strict components of $R$. More interesting is that family has an optimal element called the optimal strict component $P$ of $R$ associated to 2 . 
Let us give expressions of optimal intuitionistic fuzzy strict components $P$ of $R$ associated to $\partial$ in the general case and for the three particular cases where $\partial \in\left\{\partial_{M}, \partial_{L}\right.$, $2 \mathrm{P}\}$.

Example 4.6. (1) If $\partial=(S, T)$ satisfying $G$, then Theorem 4.5 implies that $R$ has an optimal strict component $P$ defined by, $\forall a, b \in X$,

$$
\begin{gathered}
\mu_{P}(a, b)=J_{S}\left(\mu_{R}(b, a), \mu_{R}(a, b)\right), \\
v_{P}(a, b)=I_{T}\left(v_{R}(b, a), v_{R}(a, b)\right) .
\end{gathered}
$$

(2) If $\partial=\partial_{M}=\left(S_{\mathbf{M}}, T_{\mathbf{M}}\right)$, then the optimal strict component $P$ of $R$ is defined by, $\forall a, b \in X$,

$$
\begin{aligned}
\mu_{P}(a, b) & =J_{S_{\mathrm{M}}}\left(\mu_{R}(b, a), \mu_{R}(a, b)\right) \\
& = \begin{cases}0, & \text { if } \mu_{R}(a, b) \leq \mu_{R}(b, a) \\
\mu_{R}(a, b), & \text { otherwise, }\end{cases} \\
v_{P}(a, b) & =I_{T_{\mathrm{M}}}\left(v_{R}(b, a), v_{R}(a, b)\right) \\
& = \begin{cases}1, & \text { if } v_{R}(a, b) \geq v_{R}(b, a), \\
v_{R}(a, b), & \text { otherwise. }\end{cases}
\end{aligned}
$$

This version is the one obtained by Dimitrov (see [18] or Proposition 4.1).

(3) If $\partial=\partial_{\mathrm{L}}=\left(S_{\mathrm{L}}, T_{\mathrm{L}}\right)$, then the optimal strict component $P$ of $R$ is defined by, $\forall a, b \in$ $X$,

$$
\begin{aligned}
\mu_{P}(a, b) & =J_{S_{\mathrm{L}}}\left(\mu_{R}(b, a), \mu_{R}(a, b)\right) \\
& = \begin{cases}0, & \text { if } \mu_{R}(a, b) \leq \mu_{R}(b, a) \\
\mu_{R}(a, b)-\mu_{R}(b, a), & \text { otherwise, }\end{cases} \\
v_{P}(a, b) & =I_{T_{\mathrm{L}}}\left(v_{R}(b, a), v_{R}(a, b)\right) \\
& = \begin{cases}1, & \text { if } v_{R}(a, b) \geq v_{R}(b, a), \\
1+v_{R}(a, b)-v_{R}(b, a), & \text { otherwise. }\end{cases}
\end{aligned}
$$


(4) If $\partial=\partial_{\mathrm{P}}=\left(S_{\mathbf{P}}, T_{\mathbf{P}}\right)$, then the optimal strict component $P$ of $R$ is defined by, $\forall a, b \in$ $X$,

$$
\begin{aligned}
\mu_{P}(a, b) & =J_{S_{\mathrm{P}}}\left(\mu_{R}(b, a), \mu_{R}(a, b)\right) \\
& = \begin{cases}0, & \text { if } \mu_{R}(a, b) \leq \mu_{R}(b, a), \\
\frac{\mu_{R}(a, b)-\mu_{R}(b, a)}{1-\mu_{R}(b, a)}, & \text { otherwise, }\end{cases} \\
v_{P}(a, b) & =I_{T_{\mathrm{P}}}\left(v_{R}(b, a), v_{R}(a, b)\right) \\
& = \begin{cases}1, & \text { if } v_{R}(a, b) \geq v_{R}(b, a), \\
\frac{v_{R}(a, b)}{v_{R}(b, a)}, & \text { otherwise. }\end{cases}
\end{aligned}
$$

It is interesting to give some cases of 2 where the family of intuitionistic fuzzy strict components of $R$ has a unique element (i.e., becomes the optimal intuitionistic fuzzy strict component).

The following result specifies that we have a unique intuitionistic fuzzy strict component of an IFR $R$ if $\partial$ is a strict t-conorm satisfying $G$ or $\partial=\partial \mathrm{M}$.

Corollary 4.7. Let $\partial=(S, T), R$ be an IFR; I and $P$ are two IFRs. equivalent:

If $\partial$ is a strict $t$-conorm satisfying $G$, or $\partial=\partial \mathbf{M}$, thus the two following statements are

(1) $I$ and $P$ are "indifference of $R$ " and "strict component of $R$ " associated to 2 respectively.

(2) I and $P$ are, respectively, defined by, $\forall x, y \in X$,

$$
\begin{gathered}
\mu_{I}(x, y)=\mu_{I}(y, x)=\min \left(\mu_{R}(x, y), \mu_{R}(y, x)\right), \\
v_{I}(x, y)=v_{I}(y, x)=\max \left(v_{R}(x, y), v_{R}(y, x)\right), \\
\mu_{P}(x, y)=J_{S}\left(\mu_{R}(y, x), \mu_{R}(x, y)\right), \\
v_{P}(x, y)=I_{T}\left(v_{R}(y, x), v_{R}(x, y)\right) .
\end{gathered}
$$

The following result shows that when the IFR $R$ becomes a FR, the previous theorem becomes the factorization established by Fono and Andjiga (see [7, Proposition 3, page 378]).

Corollary 4.8. Let $\partial=(S, T), R$ be an IFR; I and $P$ are two IFRs.

If $T$ and $S$ are dual and $R$ becomes $a F R$, then the two following statements are equivalent:

(1) $I$ and $P$ are "indifference of $R$ " and "strict component of $R$ " associated to 2, respectively.

(2) I and $P$ are, respectively, defined by, $\forall x, y \in X$;

(i) $\mu_{I}(x, y)=\mu_{I}(y, x)=\min \left(\mu_{R}(x, y), \mu_{R}(y, x)\right)$;

(ii) $\mu_{R}(x, y) \leq \mu_{R}(y, x) \Leftrightarrow \mu_{P}(x, y)=0$;

(iii) $\mu_{R}(x, y)>\mu_{R}(y, x) \Leftrightarrow\left(\mu_{P}(x, y)>0\right.$, and $\mu_{P}(x, y)$ is a solution of $\left.(4.9)\left(E_{1}\right)\right)$. 
In this case, "indifference of an IFR" and "strict component of an IFR" become "indifference of a FR" and "strict component of a FR" associated to $S$, respectively.

In the rest of the paper, we study two properties of a given strict component of an IFR.

In literature of (binary) crisp relations, it is well-known that the unique strict component $P$ of a given reflexive, complete, and transitive crisp relation $R$ satisfies those two interesting and usual properties, namely, pos-transitivity, that is, $\forall x, y, z \in$ $X,(x P y$ and $y P z) \Rightarrow x P z$ and negative transitivity, that is, $\forall x, y, z \in X, x P z \Rightarrow$ $(x P y$ or $y P z)$.

Fono and Andjiga [7] showed that this result is no true in the fuzzy case. More precisely, they introduced fuzzy versions of these properties (see [7, Definition 5, page 379]), showed that some strict components violate these fuzzy versions (see [7, Example 2, page 383]). They determined necessary and sufficient conditions on a reflexive, weakly complete and $T$-transitive FR $R$ such that a regular fuzzy strict component of $R$ satisfies each of these properties (see [7, Propositions 6 and 7, page 381]).

Following this line, the aim of the sequel is to (i) introduce a version of pos-transitivity for IFRs and a version of negative transitivity for IFRs and (ii) determine necessary and sufficient conditions on a given $\tau$-transitive IFR $R$ under which a strict component of $R$ satisfies the introduced properties.

\section{Properties of a Strict Component of an IFR}

\subsection{Definitions and Examples of Properties, and New Conditions on an IFR}

Definition 5.1. Let $R$ be an IFR, and let $P$ be a strict component of $R$.

(1) $P$ is pos-transitive if $\forall x, y, z \in X$,

$$
\left(\left\{\begin{array} { l } 
{ \mu _ { P } ( x , y ) > 0 } \\
{ v _ { P } ( x , y ) < 1 , }
\end{array} \{ \begin{array} { l } 
{ \mu _ { P } ( y , z ) > 0 } \\
{ v _ { P } ( y , z ) < 1 }
\end{array} ) \text { imply } \left\{\begin{array}{l}
\mu_{P}(x, z)>0 \\
v_{P}(x, z)<1
\end{array}\right.\right.\right.
$$

(2) $P$ is negative transitive if $\forall x, y, z \in X$,

$$
\left(\left\{\begin{array} { l } 
{ \mu _ { P } ( x , y ) = 0 } \\
{ v _ { P } ( x , y ) = 1 , }
\end{array} \quad \{ \begin{array} { l } 
{ \mu _ { P } ( y , z ) = 0 } \\
{ v _ { P } ( y , z ) = 1 }
\end{array} ) \text { imply } \left\{\begin{array}{l}
\mu_{P}(x, z)=0 \\
v_{P}(x, z)=1
\end{array}\right.\right.\right.
$$

Let us give the following remark on these definitions.

Remark 5.2. (1) As $P$ is regular, we can rewrite the pos-transitivity as follows: $\forall x, y, z \in X$,

$$
\begin{aligned}
& \text { (i) }\left\{\begin{array}{l}
\mu_{R}(x, y)>\mu_{R}(y, x) \\
\mu_{R}(y, z)>\mu_{R}(z, y)
\end{array} \text { imply } \mu_{R}(x, z)>\mu_{R}(z, x),\right. \\
& \text { (ii) }\left\{\begin{array}{l}
v_{R}(x, y)<v_{R}(y, x) \\
v_{R}(y, z)<v_{R}(z, y)
\end{array} \text { imply } v_{R}(x, z)<v_{R}(z, x) .\right.
\end{aligned}
$$


(2) As $P$ is regular, the negative transitivity is equivalent to the following conjunction: $\forall x, y, z \in X$,

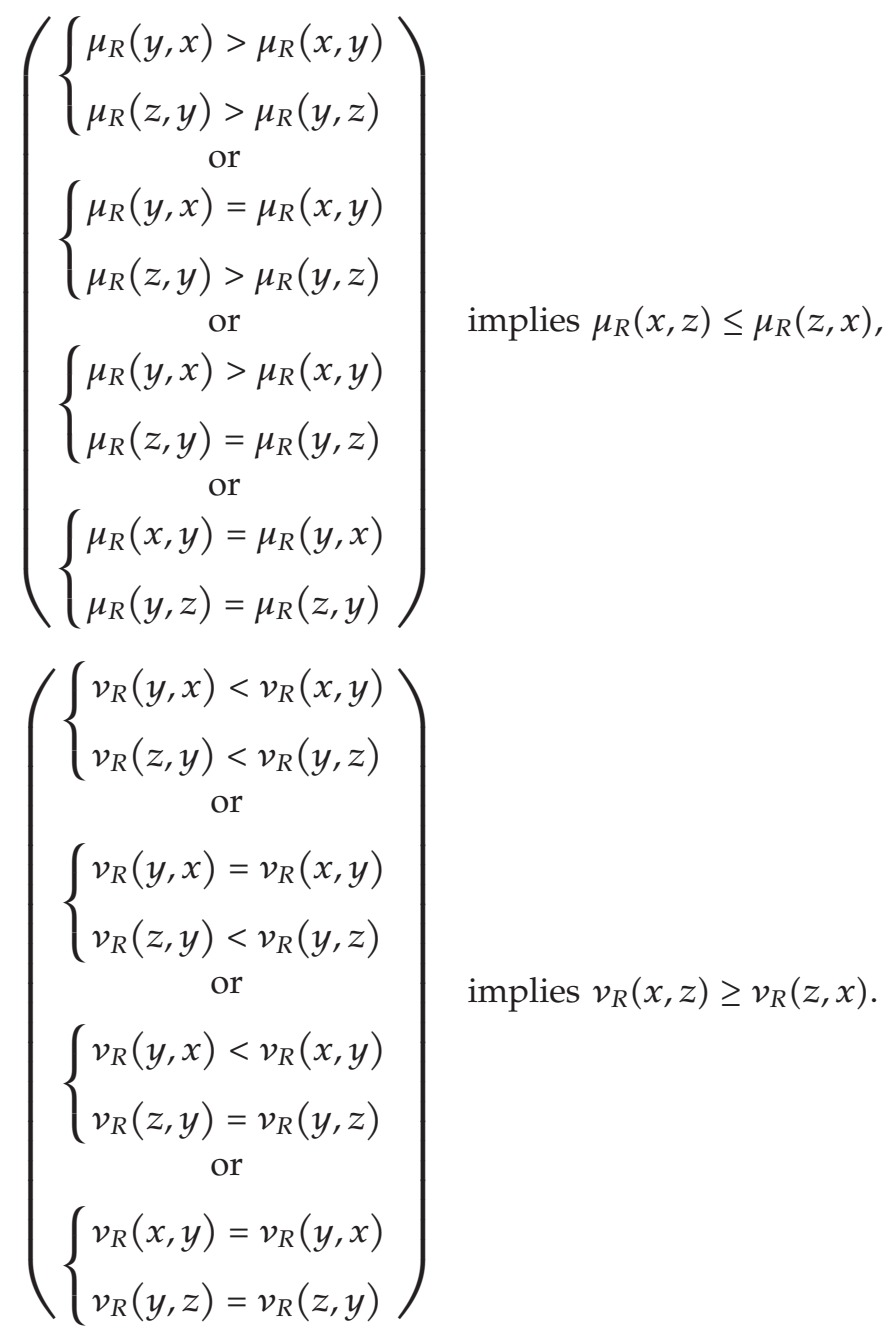

(3) Since $R$ is $\pi$-symmetric, then

$\left\{\begin{array}{l}\mu_{R}(y, x)<\mu_{R}(x, y) \\ \mu_{R}(z, y)<\mu_{R}(y, z),\end{array} \quad\left\{\begin{array}{l}\mu_{R}(y, x)=\mu_{R}(x, y) \\ \mu_{R}(z, y)<\mu_{R}(y, z),\end{array} \quad\left\{\begin{array}{l}\mu_{R}(y, x)<\mu_{R}(x, y) \\ \mu_{R}(z, y)=\mu_{R}(y, z),\end{array}\right.\right.\right.$

are equivalent to

$$
\left\{\begin{array} { l } 
{ v _ { R } ( y , x ) > v _ { R } ( x , y ) } \\
{ v _ { R } ( z , y ) > v _ { R } ( y , z ) , }
\end{array} \quad \left\{\begin{array} { l } 
{ v _ { R } ( y , x ) = v _ { R } ( x , y ) } \\
{ v _ { R } ( z , y ) > v _ { R } ( y , z ) , }
\end{array} \quad \left\{\begin{array}{l}
v_{R}(y, x)>v_{R}(x, y) \\
v_{R}(z, y)=v_{R}(y, z),
\end{array}\right.\right.\right.
$$

respectively. 
One of the main questions is to wonder if a strict component of a given IFR, which is not a FR, satisfies each of these two properties.

In the following, we justify that there exists an IFR $R$ (distinct to FRs) such that some strict components of $R$ violate each of these properties.

Example 5.3. Let $X=\{x, y, z\}$ and $\partial_{\mathrm{L}}$ (thus by Proposition 2.10, $\partial_{\mathrm{L}}$ satisfies condition $G$ ).

(1) We determine an IFR $R$ on $X$ such that there exists a strict component $P$ of $R$ which violates pos-transitivity, (i.e., $P$ satisfies: there exists $u, v, w \in X$ such that

$$
\begin{array}{r}
\left\{\begin{array} { l } 
{ \mu _ { P } ( u , v ) > 0 } \\
{ v _ { P } ( u , v ) < 1 , }
\end{array} \quad \left\{\begin{array}{l}
\mu_{P}(v, w)>0 \\
v_{P}(v, w)<1,
\end{array}\right.\right. \\
\left(\mu_{P}(u, w)=0 \text { or } v_{P}(u, w)=1\right) .
\end{array}
$$

Let $R$ be defined by, $\forall d \in X, R(d, d)=(1,0) ; R(x, y)=(0.7,0.2) ; R(y, x)=$ $(0.5,0.4) ; R(y, z)=(0.6,0.2) ; R(z, y)=(0.5,0.3) ; R(x, z)=(0.5,0.4) ; R(z, x)=(0.8,0.1)$.

Clearly, $R$ is reflexive, weakly complete, and $\pi$-symmetric. By Theorem $4.5, R$ has an optimal strict component $P_{\mathrm{L}}$ defined by (4.15).

We have: $\mu_{P_{\mathrm{L}}}(x, y)=\mu_{R}(x, y)-\mu_{R}(y, x)=0.2>0 ; \mu_{P_{\mathrm{L}}}(y, z)=\mu_{R}(y, z)-\mu_{R}(z, y)=0.1>$ $0 ; v_{P_{\mathrm{L}}}(x, y)=1+v_{R}(x, y)-v_{R}(y, x)=0.8<1$ and $v_{P_{\mathrm{L}}}(y, z)=1+v_{R}(y, z)-v_{R}(z, y)=0.9<1$, whereas $\mu_{P_{\mathrm{L}}}(x, z)=0$ and $v_{P_{\mathrm{L}}}(x, z)=1$.

In other words, $x$ is strictly preferred to $\mathrm{y}$ ( since $\mu_{P_{\mathrm{L}}}(x, y)>0$ and $v_{P_{\mathrm{L}}}(x, y)<1$ ), and $y$ is strictly preferred to $z$ (since $\mu_{P_{\mathrm{L}}}(y, z)>0$ and $v_{P_{\mathrm{L}}}(y, z)<1$ ), but $x$ is not strictly preferred to $z$ (since $\mu_{P_{\mathrm{L}}}(x, z)=0$ and $v_{P_{\mathrm{L}}}(x, z)=1$ ).

Hence $P_{\mathbf{L}}$ violates pos-transitivity.

(2) We determine an IFR $R$ on $X$ such that there exists a strict component $P$ of $R$ which violates negative transitivity, (i.e., $P$ satisfies: there exists $u, v, w \in X$ such that

$$
\begin{gathered}
\left\{\begin{array} { l } 
{ \mu _ { P } ( u , v ) = 0 } \\
{ v _ { P } ( u , v ) = 1 , }
\end{array} \quad \left\{\begin{array}{l}
\mu_{P}(v, w)=0 \\
v_{P}(v, w)=1,
\end{array}\right.\right. \\
\left(\mu_{P}(u, w)>0 \text { or } v_{P}(u, w)<1\right) .
\end{gathered}
$$

Let $R$ be defined by, $\forall d \in X, R(d, d)=(1,0) ; R(x, y)=(0.5,0.4) ; R(y, x)=$ $(0.7,0.2) ; R(y, z)=(0.4,0.5) ; R(z, y)=(0.8,0.1) ; R(x, z)=(0.6,0.1) ; R(z, x)=(0.4,0.3)$.

Clearly, $R$ is reflexive, weakly complete, and $\pi$-symmetric. By Theorem $4.5, R$ has an optimal strict component $P_{\mathrm{L}}$ defined by (4.15).

We have $\mu_{P_{\mathrm{L}}}(x, y)=\mu_{P_{\mathrm{L}}}(y, z)=0$ and $v_{P_{\mathrm{L}}}(x, y)=v_{P_{\mathrm{L}}}(y, z)=1$, whereas $\mu_{P_{\mathrm{L}}}(x, z)=$ $\mu_{R}(x, z)-\mu_{R}(z, x)=0.2>0$, and $v_{P_{\mathrm{L}}}(x, z)=1+v_{R}(x, z)-v_{R}(z, x)=0.8<1$.

In other words, $x$ is strictly preferred to $z$ (since $\mu_{P_{\mathrm{L}}}(x, z)>0$ and $v_{P_{\mathrm{L}}}(x, z)<1$ ), but $x$ is not strictly preferred to $y$ (since $\mu_{P_{\mathrm{L}}}(x, y)=0$ and $v_{P_{\mathrm{L}}}(x, y)=1$ ), and $y$ is not strictly preferred to $z$ (since $\mu_{P_{\mathrm{L}}}(y, z)=0$ and $v_{P_{\mathrm{L}}}(y, z)=1$ ).

Hence $P_{\mathbf{L}}$ violates negative transitivity. 
This enables us to determine necessary and sufficient conditions on an IFR $R$ such that a given strict component $P_{R}$ of $R$ is pos-transitive and negative transitive. For that we introduce the following conditions.

Definition 5.4. Let $R$ be an IFR.

(1) (i) $R$ satisfies condition $C_{1}^{\mu_{R}}$ if $\forall x, y, z \in X$,

$$
\begin{aligned}
& \mu_{R}(y, x)<\mu_{R}(x, y) \\
& \mu_{R}(z, y)<\mu_{R}(y, z)
\end{aligned} \Longrightarrow\left(\left\{\begin{array}{l}
\mu_{R}(x, z) \in\left[\alpha_{2}(x, y, z), \alpha_{3}(x, y, z)\right] \\
\mu_{R}(z, x) \in\left[\alpha_{2}(x, y, z), \alpha_{3}(x, y, z)\right]
\end{array} \Longrightarrow \mu_{R}(z, x)<\mu_{R}(x, z)\right)\right.
$$

(ii) $R$ satisfies condition $C_{1}^{v_{R}}$ if $\forall x, y, z \in X$,

$$
\begin{aligned}
& v_{R}(y, x)>v_{R}(x, y) \\
& v_{R}(z, y)>v_{R}(y, z)
\end{aligned} \Longrightarrow\left(\left\{\begin{array}{l}
v_{R}(x, z) \in\left[\beta_{3}(x, y, z), \beta_{2}(x, y, z)\right] \\
v_{R}(z, x) \in\left[\beta_{3}(x, y, z), \beta_{2}(x, y, z)\right]
\end{array} \Longrightarrow v_{R}(z, x)>v_{R}(x, z)\right)\right.
$$

(2) (i) $R$ satisfies condition $C_{2}^{\mu_{R}}$ if $\forall x, y, z \in X$,

$$
\begin{aligned}
& \left(\left\{\begin{array}{l}
\mu_{R}(y, x)=\mu_{R}(x, y) \\
\mu_{R}(z, y)<\mu_{R}(y, z)
\end{array} \text { or }\left\{\begin{array}{l}
\mu_{R}(y, x)<\mu_{R}(x, y) \\
\mu_{R}(z, y)=\mu_{R}(y, z)
\end{array}\right)\right.\right.
\end{aligned}
$$

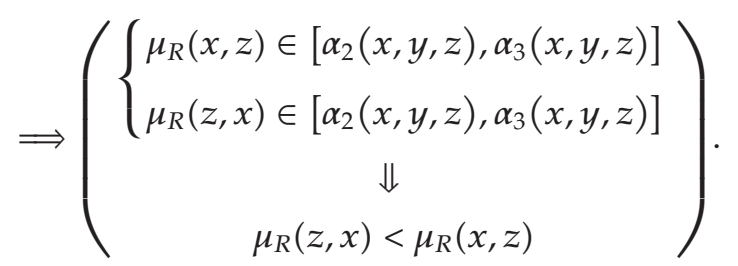

(ii) $R$ satisfies condition $C_{2}^{\nu_{R}}$ if $\forall x, y, z \in X$,

$$
\begin{gathered}
\left(\left\{\begin{array}{l}
v_{R}(y, x)=v_{R}(x, y) \\
v_{R}(z, y)>v_{R}(y, z)
\end{array} \text { or }\left\{\begin{array}{l}
v_{R}(y, x)>v_{R}(x, y) \\
v_{R}(z, y)=v_{R}(y, z)
\end{array}\right)\right.\right. \\
\Longrightarrow\left(\begin{array}{c}
v_{R}(x, z) \in\left[\beta_{3}(x, y, z), \beta_{2}(x, y, z)\right] \\
v_{R}(z, x) \in\left[\beta_{3}(x, y, z), \beta_{2}(x, y, z)\right] \\
\Downarrow
\end{array}\right) . \\
v_{R}(z, x)>v_{R}(x, z)
\end{gathered}
$$

The next result shows that a strongly complete IFR $R$ satisfies the previous conditions. 
Proposition 5.5. Let $R$ be an IFR.

If $R$ is strongly complete, then $R$ satisfies conditions $C_{1}^{\mu_{R}}, C_{1}^{v_{R}}, C_{2}^{\mu_{R}}$, and $C_{2}^{v_{R}}$.

\subsection{Characterization of Some Properties of a Strict Component of an IFR}

We now establish, using $C_{1}^{\mu_{R}}$ and $C_{1}^{\nu_{R}}$, our second main result which determines all $\tau$ transitive IFRs whose strict components are pos-transitive.

Theorem 5.6. Let $R$ be a $\tau$-transitive IFR, and let $P$ be a strict component of $R$. Then

$$
\left(\begin{array}{c}
R \text { satisfies condition } C_{1}^{\mu_{R}} \\
\text { or } \\
R \text { satisfies condition } C_{1}^{v_{R}}
\end{array}\right) \Longleftrightarrow(P \text { is pos-transitive })
$$
follows:

In the particular case where $R$ is strongly complete, the previous result becomes as

Corollary 5.7. Let $R$ be a $\tau$-transitive IFR, and let $P$ be a strict component of $R$.

If $R$ is strongly complete, then $P$ is pos-transitive.

Given a $\tau$-transitive IFR $R$, the next result establishes an equivalence between conditions $C_{2}^{\mu_{R}}$ and $C_{2}^{\nu_{R}}$, and two properties of $P$.

Lemma 5.8. Let $R$ be a $\tau$-transitive IFR, and let $P$ be a strict component of $R$.

The two following statements are equivalent:

(1) R satisfies condition $C_{2}^{\mu_{R}}$ or condition $C_{2}^{\nu_{R}}$;

(2)

$$
\forall x, y, z \in X
$$

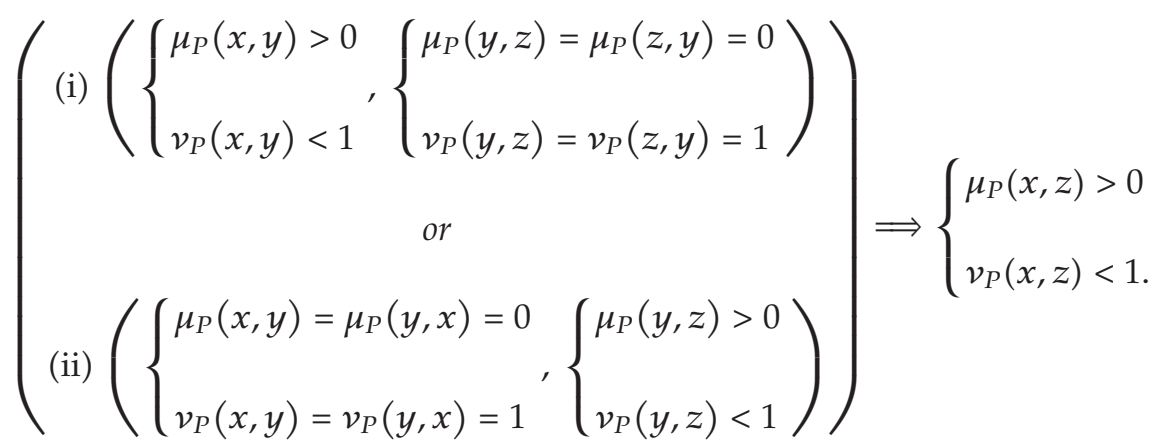


It is important to notice that as $P$ is regular and $R$ is $\pi$-symmetric, we can rewrite (5.15) as follows:

$$
\left.\begin{array}{c}
\text { (i) }\left(\left\{\begin{array}{c}
\mu_{R}(y, x)=\mu_{R}(x, y) \\
\mu_{R}(z, y)<\mu_{R}(y, z)
\end{array} \text { or }\left\{\begin{array}{c}
\mu_{R}(y, x)<\mu_{R}(x, y) \\
\mu_{R}(z, y)=\mu_{R}(y, z)
\end{array}\right) \Longrightarrow \mu_{R}(x, z)>\mu_{R}(z, x)\right.\right. \\
\text { or }
\end{array}\right) .
$$

The third and last main result of our paper determines all $\tau$-transitive IFRs whose strict components are negative transitive.

Theorem 5.9. Let $R$ be a $\tau$-transitive IFR, and let $P$ be a strict component of $R$. Then

$$
\left(\begin{array}{c}
R \text { satisfies conditions } C_{1}^{\mu_{R}} \text { and } C_{2}^{\mu_{R}} \\
\text { or } \\
R \text { satisfies conditions } C_{1}^{v_{R}} \text { and } C_{2}^{v_{R}}
\end{array}\right) \Longleftrightarrow(P \text { is negative transitive }) .
$$
follows:

In the particular case where $R$ is strongly complete, the previous result becomes as

Corollary 5.10. Let $R$ be a $\tau$-transitive IFR, and let $P$ be a strict component of $R$. If $R$ is strongly complete, then $P$ is negative transitive.

\section{Concluding Remarks}

In this paper, we establish a characterization of the $\tau$-transitivity of $R$. We also establish a general factorization of an intuitionistic fuzzy binary relation when the union is defined by a continuous t-representable intuitionistic fuzzy t-conorm satisfying condition $G$. This factorization gives a family of regular strict components. Furthermore, given an IFR $R$, we introduce two conditions $C_{1}^{\mu_{R}}$ and $C_{2}^{\mu_{R}}$ (or equivalently $C_{1}^{\nu_{R}}$ and $C_{2}^{\nu_{R}}$ ). And we show that, when $R$ is $\tau$-transitive, these conditions are necessary and sufficient to obtain pos-transitivity and negative transitivity of a given strict component of $R$.

An open problem is to apply these results especially in social choice theory when individual and social preferences are modelled by reflexive, weakly complete, and $\tau$ transitive IFRs. Another open problem is to study the properties of the class of continuous $\mathrm{t}$-representable intuitionistic fuzzy t-conorms satisfying condition $G$. 


\section{Appendix}

\section{The Proofs of our Results}

Proof of Proposition 2.7. (1) Let $a, b \in[0,1]$. Since $T(a, b) \leq 1-S(1-a, 1-b)$, then $\{t \in[0,1], 1-$ $S(1-a, 1-t) \leq b\} \subseteq\{t \in[0,1], T(a, t) \leq b\}$. Set $t^{\prime}=1-t$, we have $I_{T}(a, b) \geq \max \{t \in$ $[0,1], 1-S(1-a, 1-t) \leq b\}=\max \{t \in[0,1], S(1-a, 1-t) \geq 1-b\}=\max \left\{1-t^{\prime} \in\right.$ $\left.[0,1], S\left(1-a, t^{\prime}\right) \geq 1-b\right\}=1-\min \left\{t^{\prime} \in[0,1], S\left(1-a, t^{\prime}\right) \geq 1-b\right\}=1-J_{S}(1-a, 1-b)$.

(2) The proof is obvious.

Proof of Lemma 2.8. (1) Let $x=\left(x_{1}, x_{2}\right), y=\left(y_{1}, y_{2}\right) \in L^{*}$. Set $A=\left\{z=\left(z_{1}, z_{2}\right) \in L^{*}, y_{1} \leq\right.$ $S\left(x_{1}, z_{1}\right)$ and $\left.y_{2} \geq T\left(x_{2}, z_{2}\right)\right\} \neq \emptyset$. That is to show that $\inf A=\left(\min \left\{z_{1} \in[0,1], y_{1} \leq\right.\right.$ $\left.\left.S\left(x_{1}, z_{1}\right)\right\}, \min \left(\max \left\{z_{2} \in[0,1], T\left(x_{2}, z_{2}\right) \leq y_{2}\right\}, 1-\min \left\{z_{1} \in[0,1], y_{1} \leq S\left(x_{1}, z_{1}\right)\right\}\right)\right)$.

Since $A \subseteq L^{*}$ and $\left(L^{*}, L_{L^{*}}\right)$ is a complete lattice, and $T$ and $S$ are continuous functions on the compact $[0,1] \times[0,1]$, then the definition of lower limit in $L^{*}$ gives

$$
\begin{aligned}
\inf A= & \left(\inf \left\{z_{1} \in[0,1], \exists t_{1} \in[0,1],\left(z_{1}, t_{1}\right) \in A\right\}, \sup \left\{z_{2} \in[0,1], \exists t_{2} \in[0,1],\left(t_{2}, z_{2}\right) \in A\right\}\right) \\
= & \inf \left\{z_{1} \in[0,1], \exists t_{1} \in[0,1],\left\{\begin{array}{l}
y_{1} \leq S\left(x_{1}, z_{1}\right) \\
y_{2} \geq T\left(x_{2}, t_{1}\right) \\
z_{1}+t_{1} \leq 1
\end{array}\right\},\right. \\
& \sup \left\{z_{2} \in[0,1], \exists t_{2} \in[0,1],\left\{\begin{array}{l}
y_{1} \leq S\left(x_{1}, t_{2}\right) \\
y_{2} \geq T\left(x_{2}, z_{2}\right) \\
z_{2}+t_{2} \leq 1
\end{array}\right\}\right) \\
= & \min \left\{\begin{array}{l}
z_{1} \in[0,1], \exists t_{1} \in[0,1],\left\{\begin{array}{l}
y_{1} \leq S\left(x_{1}, z_{1}\right) \\
y_{2} \geq T\left(x_{2}, t_{1}\right) \\
z_{1}+t_{1} \leq 1
\end{array}\right\}, \\
\left.z_{2} \in[0,1], \exists t_{2} \in[0,1],\left\{\begin{array}{l}
y_{1} \leq S\left(x_{1}, t_{2}\right) \\
y_{2} \geq T\left(x_{2}, z_{2}\right) \\
z_{2}+t_{2} \leq 1
\end{array}\right\}\right) .
\end{array}\right.
\end{aligned}
$$

Otherwise, with the second result of Proposition 2.1, we have

$$
\begin{aligned}
& \left\{\begin{array}{l}
y_{1} \leq S\left(x_{1}, z_{1}\right) \\
y_{2} \geq T\left(x_{2}, t_{1}\right) \\
z_{1}+t_{1} \leq 1
\end{array}\right. \\
& \left\{\begin{array} { l } 
{ y _ { 1 } \leq S ( x _ { 1 } , t _ { 2 } ) } \\
{ y _ { 2 } \geq T ( x _ { 2 } , z _ { 2 } ) } \\
{ z _ { 2 } + t _ { 2 } \leq 1 }
\end{array} \Longleftrightarrow \left\{\begin{array}{l}
z_{1} \in\left[J_{S}\left(x_{1}, y_{1}\right), 1\right] \\
t_{1} \in\left[0, I_{T}\left(x_{2}, y_{2}\right)\right] \\
z_{1}+t_{1} \leq 1,
\end{array}\right.\right. \\
&
\end{aligned}
$$


We distinguish three cases.

(i) If $x_{1}<y_{1}$ and $x_{2} \leq y_{2}$, then with the third result of Proposition 2.1, we have $J_{S}\left(x_{1}, y_{1}\right)>0$ and $I_{T}\left(x_{2}, y_{2}\right)=1$. This implies $I_{T}\left(x_{2}, y_{2}\right)>1-J_{S}\left(x_{1}, y_{1}\right)$. Thus,

$$
\begin{gathered}
\min \left\{z_{1} \in[0,1], \exists t_{1} \in[0,1],\left\{\begin{array}{l}
y_{1} \leq S\left(x_{1}, z_{1}\right) \\
y_{2} \geq T\left(x_{2}, t_{1}\right) \\
z_{1}+t_{1} \leq 1
\end{array}\right\}=J_{S}\left(x_{1}, y_{1}\right),\right. \\
\max \left\{z_{2} \in[0,1], \exists t_{2} \in[0,1],\left\{\begin{array}{l}
y_{1} \leq S\left(x_{1}, t_{2}\right) \\
y_{2} \geq T\left(x_{2}, z_{2}\right) \\
z_{2}+t_{2} \leq 1
\end{array}\right\}=1-J_{S}\left(x_{1}, y_{1}\right) .\right.
\end{gathered}
$$

Hence $J_{2}(x, y)=\left(J_{S}\left(x_{1}, y_{1}\right), 1-J_{S}\left(x_{1}, y_{1}\right)\right)$.

(ii) If $\left(x_{1} \geq y_{1}\right.$ and $\left.x_{2} \leq y_{2}\right)$ or $\left(x_{1} \geq y_{1}\right.$ and $\left.x_{2}>y_{2}\right)$, then with the third result of Proposition 2.1, it is easy to show that

$$
\begin{gathered}
\min \left\{z_{1} \in[0,1], \exists t_{1} \in[0,1],\left\{\begin{array}{l}
y_{1} \leq S\left(x_{1}, z_{1}\right) \\
y_{2} \geq T\left(x_{2}, t_{1}\right) \\
z_{1}+t_{1} \leq 1
\end{array}\right\}=J_{S}\left(x_{1}, y_{1}\right),\right. \\
\max \left\{z_{2} \in[0,1], \exists t_{2} \in[0,1],\left\{\begin{array}{l}
y_{1} \leq S\left(x_{1}, t_{2}\right) \\
y_{2} \geq T\left(x_{2}, z_{2}\right) \\
z_{2}+t_{2} \leq 1
\end{array}\right\}=I_{T}\left(x_{2}, y_{2}\right) \leq 1-J_{S}\left(x_{1}, y_{1}\right) .\right.
\end{gathered}
$$

Hence $J_{2}(x, y)=\left(J_{S}\left(x_{1}, y_{1}\right), I_{T}\left(x_{2}, y_{2}\right)\right)$.

(iii) If $x_{1}<y_{1}$ and $x_{2}>y_{2}$, then with the third result of Proposition 2.1, $J_{S}\left(x_{1}, y_{1}\right)>0$, and $I_{T}\left(x_{2}, y_{2}\right)<1$. We distinguish two cases.

(a) If $I_{T}\left(x_{2}, y_{2}\right) \leq 1-J_{S}\left(x_{1}, y_{1}\right)$, thus

$$
\begin{gathered}
\min \left\{z_{1} \in[0,1], \exists t_{1} \in[0,1],\left\{\begin{array}{l}
y_{1} \leq S\left(x_{1}, z_{1}\right) \\
y_{2} \geq T\left(x_{2}, t_{1}\right) \\
z_{1}+t_{1} \leq 1
\end{array}\right\}=J_{S}\left(x_{1}, y_{1}\right),\right. \\
\max \left\{z_{2} \in[0,1], \exists t_{2} \in[0,1],\left\{\begin{array}{l}
y_{1} \leq S\left(x_{1}, t_{2}\right) \\
y_{2} \geq T\left(x_{2}, z_{2}\right) \\
z_{2}+t_{2} \leq 1
\end{array}\right\}=I_{T}\left(x_{2}, y_{2}\right) .\right.
\end{gathered}
$$

Hence $J_{2}(x, y)=\left(J_{S}\left(x_{1}, y_{1}\right), I_{T}\left(x_{2}, y_{2}\right)\right)$. 
(b) If $I_{T}\left(x_{2}, y_{2}\right)>1-J_{S}\left(x_{1}, y_{1}\right)$, thus

$$
\begin{gathered}
\min \left\{z_{1} \in[0,1], \exists t_{1} \in[0,1],\left\{\begin{array}{l}
y_{1} \leq S\left(x_{1}, z_{1}\right) \\
y_{2} \geq T\left(x_{2}, t_{1}\right) \\
z_{1}+t_{1} \leq 1
\end{array}\right\}=J_{S}\left(x_{1}, y_{1}\right),\right. \\
\max \left\{z_{2} \in[0,1], \exists t_{2} \in[0,1],\left\{\begin{array}{l}
y_{1} \leq S\left(x_{1}, t_{2}\right) \\
y_{2} \geq T\left(x_{2}, z_{2}\right) \\
z_{2}+t_{2} \leq 1
\end{array}\right\}=1-J_{S}\left(x_{1}, y_{1}\right) .\right.
\end{gathered}
$$

Hence $J_{2}(x, y)=\left(J_{S}\left(x_{1}, y_{1}\right), 1-J_{S}\left(x_{1}, y_{1}\right)\right)$.

Finally, we obtain $J_{2}(x, y)=\left(J_{S}\left(x_{1}, y_{1}\right), \min \left(I_{T}\left(x_{2}, y_{2}\right), 1-J_{S}\left(x_{1}, y_{1}\right)\right)\right)$.

(2) The proof of the second result is analogous to the previous one.

Proof of Proposition 2.10. (1) Let $\left(a_{1}, a_{2}\right),\left(b_{1}, b_{2}\right) \in L^{*}$ such that

$$
\begin{gathered}
a_{1}>b_{1}, \\
a_{2}<b_{2}, \\
a_{1}+a_{2}=b_{1}+b_{2} .
\end{gathered}
$$

(i) Since $\partial_{0}^{F}=\partial_{\mathrm{M}}, \partial_{1}^{F}=\partial_{\mathrm{p}}$ and $\partial_{\infty}^{F}=\partial_{\mathrm{L}}$, with Example 2.5, it is obvious to show that $\forall l \in\{0,1,+\infty\}, \partial_{l}^{F}$ satisfies condition $G$.

(ii) Assume that $l \in] 0,1\left[\right.$, and let us show that $\partial_{l}^{F}=\left(S_{F}^{l}, T_{F}^{l}\right)$ satisfies condition $G$, that is, $I_{T}\left(b_{2}, a_{2}\right)+J_{S}\left(b_{1}, a_{1}\right) \leq 1$. It suffices to show that $\left.\forall l \in\right] 0,1\left[, \log _{l}\left(1+\left((l-1)\left(l^{a_{2}}-\right.\right.\right.\right.$ $\left.1)) /\left(l^{b_{2}}-1\right)\right) \leq \log _{l}\left(1+(l-1)\left(l^{1-a_{1}}-1\right) /\left(l^{1-b_{1}}-1\right)\right)$. Set $c=a_{1}+a_{2}=b_{1}+b_{2}$.

(a) Since $a_{1}>b_{1}$ and the mapping $l^{t}$ is decreasing, then $l^{a_{1}} \leq l^{b_{1}}$, that is, $l^{-b_{1}} \leq l^{-a_{1}}$.

(b) Since $l^{c}-l \geq 0$, we obtain $l^{-b_{1}}\left(l^{c}-l\right) \leq l^{-a_{1}}\left(l^{c}-l\right)$, that is, $l^{b_{2}}+l^{1-a_{1}} \leq l^{a_{2}}+l^{1-b_{1}}$.

(c) Since $a_{2}-b_{1}=b_{2}-a_{1}$, the previous inequality becomes $l^{1+a_{2}-b_{1}}-l^{a_{2}}-l^{1-b_{1}}+1 \leq$ $l^{1+b_{2}-a_{1}}-l^{b_{2}}-l^{1-a_{1}}+1$, that is, $\left(l^{a_{2}}-1\right)\left(l^{1-b_{1}}-1\right) \leq\left(l^{b_{2}}-1\right)\left(l^{1-a_{1}}-1\right)$, that is, $\left(l^{a_{2}}-1\right) /\left(l^{b_{2}}-1\right) \leq\left(l^{1-a_{1}}-1\right) /\left(l^{1-b_{1}}-1\right)$, that is, $1+(l-1)\left(l^{a_{2}}-1\right) /\left(l^{b_{2}}-1\right) \geq$ $1+(l-1)\left(l^{1-a_{1}}-1\right) /\left(l^{1-b_{1}}-1\right)$. And we have $\log _{l}\left(1+(l-1)\left(l^{a_{2}}-1\right) /\left(l^{b_{2}}-1\right)\right) \leq$ $\log _{l}\left(1+(l-1)\left(l^{1-a_{1}}-1\right) /\left(l^{1-b_{1}}-1\right)\right)$.

(iii) The proof of the case $l \in] 1,+\infty[$ is similar to theprevious one. 
(2) Let $\partial^{\prime}$ be a restriction of $\partial=(S, T)$ on $L_{1}^{*}=\left\{\left(x_{1}, x_{2}\right) \in L^{*}, x_{1}+x_{2}=1\right\}$. Assume that $S$ and $T$ are dual and show that $\partial^{\prime}$ satisfies $G$. Let $\left(a_{1}, a_{2}\right),\left(b_{1}, b_{2}\right) \in L_{1}^{*}$ such that

$$
\begin{gathered}
a_{1}>b_{1}, \\
a_{2}<b_{2}, \\
a_{1}+a_{2}=b_{1}+b_{2}=1 .
\end{gathered}
$$

Let us show that $I_{T}\left(b_{2}, a_{2}\right)+J_{S}\left(b_{1}, a_{1}\right) \leq 1$.

Since $a_{1}+a_{2}=b_{1}+b_{2}=1$ and $S$ are $T$ are dual, then $I_{T}\left(b_{2}, a_{2}\right)=I_{T}\left(1-b_{1}, 1-a_{1}\right)$. Thus, $I_{T}\left(b_{2}, a_{2}\right)+J_{S}\left(b_{1}, a_{1}\right)=I_{T}\left(1-b_{1}, 1-a_{1}\right)+J_{S}\left(b_{1}, a_{1}\right)=1$. Hence the result.

Proof of Proposition 3.4. Suppose that $R$ is $(S, T)$-complete and let us show that $R$ is $D$ complete.

Let $(x, y) \in X \times X$ such that $\left(\mu_{R}(x, y), v_{R}(x, y)\right)=(0,1)$. Thus $S\left(0, \mu_{R}(y, x)\right)=1$ and $T\left(1, v_{R}(y, x)\right)=0$. Since $S\left(0, \mu_{R}(y, x)\right)=\mu_{R}(y, x)$ and $T\left(1, v_{R}(y, x)\right)=v_{R}(y, x)$, we have $\mu_{R}(y, x)=1$ and $v_{R}(y, x)=0$. Hence $\mu_{R}(y, x)>0$ and $v_{R}(y, x)<1$.

Proof of Proposition 3.9. For all $x, y, z \in X$, we have the following.

(1) Let us show that $\left(\alpha_{1}(x, y, z), \beta_{1}(x, y, z)\right) \in L^{*}$. In fact, $\left(\alpha_{1}(x, y, z), \beta_{1}(x, y, z)\right)$ $=\left(T\left(\mu_{R}(z, y), \mu_{R}(y, x)\right), S\left(v_{R}(z, y), v_{R}(y, x)\right)\right)=\tau\left[\left(\mu_{R}(z, y), v_{R}(z, y)\right),\left(\mu_{R}(y, x)\right.\right.$, $\left.\left.v_{R}(y, x)\right)\right] \in L^{*}$ since $\tau$ is a t-norm on $L^{*}$.

(a) Let us show that $\left(\alpha_{3}(x, y, z), \beta_{3}(x, y, z)\right) \in L^{*}$. In fact, $\left(\alpha_{3}(x, y, z), \beta_{3}(x, y, z)\right)=$ $\min \left(I_{\tau}\left[\left(\mu_{R}(y, z), v_{R}(y, z)\right),\left(\mu_{R}(y, x), v_{R}(y, x)\right)\right], I_{\tau}\left[\left(\mu_{R}(x, y), v_{R}(x, y)\right),\left(\mu_{R}(z\right.\right.\right.$, $\left.\left.\left.y), v_{R}(z, y)\right)\right]\right)$. Since, $I_{\tau}\left[\left(\mu_{R}(y, z), v_{R}(y, z)\right),\left(\mu_{R}(y, x), v_{R}(y, x)\right)\right] \in L^{*}, I_{\tau}\left[\left(\mu_{R}(x\right.\right.$, $\left.\left.y), v_{R}(x, y)\right),\left(\mu_{R}(z, y), v_{R}(z, y)\right)\right] \in L^{*}$ and $\operatorname{Min}=(\min , \max )$ is a t-norm in $L^{*}$, then $\left(\alpha_{3}(x, y, z), \beta_{3}(x, y, z)\right) \in L^{*}$.

(b) The proofs of the assertions $\left(\alpha_{2}(x, y, z), \beta_{2}(x, y, z)\right) \in L^{*}$ and $\left(\alpha_{4}(x, y, z)\right.$, $\left.\beta_{4}(x, y, z)\right) \in L^{*}$ are analogous to the previous ones.

(2) The proof of the last result is deduced from the second result of Lemma 2.8.

Proof of Corollary 3.11. Assume that $R$ is strongly complete.

(1) Assume also that $R$ satisfies (3.9). And in the three cases, we have $\mu_{R}(x, y)=$ $\mu_{R}(y, z)=1$, and $v_{R}(x, y)=v_{R}(y, z)=0$. This implies $J_{S}\left(v_{R}(z, y), v_{R}(x, y)\right)=$ $J_{S}\left(v_{R}(y, x), v_{R}(y, z)\right)=0, J_{S}\left(v_{R}(x, y), v_{R}(z, y)\right)=v_{R}(z, y), J_{S}\left(v_{R}(y, z), v_{R}(y, x)\right)=$ $v_{R}(y, x)$ and $I_{T}\left(\mu_{R}(z, y), \mu_{R}(x, y)\right)=I_{T}\left(\mu_{R}(y, x), \mu_{R}(y, z)\right)=1, I_{T}\left(\mu_{R}(x, y)\right.$, $\left.\mu_{R}(z, y)\right)=\mu_{R}(z, y), I_{T}\left(\mu_{R}(y, z), \mu_{R}(y, x)\right)=\mu_{R}(y, x)$. And we obtain $\alpha_{2}(x, y, z)=$ $\alpha_{4}(x, y, z)=1$, and $\alpha_{3}(x, y, z)=\min \left(\mu_{R}(y, x), \mu_{R}(z, y)\right)$. Thus $\alpha_{1}(x, y, z)<1$ and $\alpha_{3}(x, y, z)<1$.

(2) Assume that $R$ satisfies (3.11). Thus $v_{R}(x, y)=v_{R}(y, z)=0$ and $\left(v_{R}(y, x)>0\right.$ or $\left.v_{R}(z, y)>0\right)$. Thus, $\beta_{2}(x, y, z)=S\left(v_{R}(x, y), v_{R}(y, z)\right)=S(0,0)=0$.

Assume to the contrary that $\beta_{1}(x, y, z)=0$. We have $\max \left(v_{R}(z, y), v_{R}(y, x)\right) \leq S\left(v_{R}(z, y)\right.$, $\left.v_{R}(y, x)\right)=\beta_{1}(x, y, z)=0$. Hence $v_{R}(y, x)=0$ and $v_{R}(z, y)=0$. This contradicts the assertion $\left(v_{R}(y, x)>0\right.$ or $\left.v_{R}(z, y)>0\right)$. 
Let us show that $\beta_{3}(x, y, z)=\max \left(v_{R}(y, x), v_{R}(z, y)\right)$ and $\beta_{4}(x, y, z)=\max (0,0)=0$. Proposition 3.9 implies that

$$
\begin{aligned}
& \beta_{3}(x, y, z)=\max \left(J_{S}\left(0, v_{R}(y, x)\right), J_{S}\left(0, v_{R}(z, y)\right)\right), \\
& \beta_{4}(x, y, z)=\max \left(J_{S}\left(v_{R}(y, x), 0\right), J_{S}\left(v_{R}(z, y), 0\right)\right) .
\end{aligned}
$$

By definition, $J_{S}\left(0, v_{R}(y, x)\right)=\min \left\{t \in[0,1] / S(0, t) \geq v_{R}(y, x)\right\}=\min \{t \in[0,1] / t \geq$ $\left.v_{R}(y, x)\right\}=\min \left[v_{R}(y, x), 1\right]=v_{R}(y, x)$ and $J_{S}\left(v_{R}(y, x), 0\right)=\min \left\{t \in[0,1] / S\left(v_{R}(y, x), t\right) \geq\right.$ $0\}=\min [0,1]=0$.

Analogously, $J_{S}\left(0, v_{R}(z, y)\right)=v_{R}(z, y)$ and $\left.J_{S}\left(v_{R}(z, y), 0\right)\right)=0$. Thus $\beta_{3}(x, y, z)=$ $\max \left(v_{R}(y, x), v_{R}(z, y)\right)$, and $\beta_{4}(x, y, z)=\max (0,0)=0$.

Proof of Corollary 3.12. Assume that $T$ and $S$ are dual and $R$ is a FR. Let $x, y, z \in X$.

(1) (i) Let us show that $\beta_{1}(x, y, z)=1-\alpha_{1}(x, y, z)$.

By definition, $\beta_{1}(x, y, z)=S\left(v_{R}(z, y), v_{R}(y, x)\right)$ and $\alpha_{1}(x, y, z)=T\left(\mu_{R}(z, y), \mu_{R}(y, x)\right)$. Since $R$ is a FR, we have $v_{R}(z, y)=1-\mu_{R}(z, y)$ and $v_{R}(y, x)=1-\mu_{R}(y, x)$. Otherwise, since $T$ and $S$ are dual, we have $S\left(v_{R}(z, y), v_{R}(y, x)\right)=1-T\left(1-v_{R}(z, y), 1-v_{R}(y, x)\right)$. Thus $\beta_{1}(x, y, z)=$ $1-T\left(1-\left(1-\mu_{R}(z, y)\right), 1-\left(1-\mu_{R}(y, x)\right)\right)=1-T\left(\mu_{R}(z, y), \mu_{R}(y, x)\right)=1-\alpha_{1}(x, y, z)$. Hence the result.

(ii) The proof of equality $\beta_{2}(x, y, z)=1-\alpha_{2}(x, y, z)$ is analogous to the previous one.

(iii) Let us show that $\beta_{3}(x, y, z)=1-\alpha_{3}(x, y, z)$.

With the previous proposition, $\beta_{3}(x, y, z)=\max \left(J_{S}\left(v_{R}(y, z), v_{R}(y, x)\right), J_{S}\left(v_{R}(x, y), v_{R}(z, y)\right)\right)$. Since $R$ is a FR, we have $v_{R}(y, z)=1-\mu_{R}(y, z), v_{R}(z, y)=1-\mu_{R}(z, y), v_{R}(y, x)=1-\mu_{R}(y, x)$, and $v_{R}(x, y)=1-\mu_{R}(x, y)$. Otherwise, since $T$ and $S$ are dual, we have $J_{S}\left(v_{R}(y, z), v_{R}(y, x)\right)=$ $1-I_{T}\left(1-v_{R}(y, z), 1-v_{R}(y, x)\right)$ and $J_{S}\left(v_{R}(x, y), v_{R}(z, y)\right)=1-I_{T}\left(1-v_{R}(x, y), 1-v_{R}(z, y)\right)$. Thus $\beta_{3}(x, y, z)=\max \left(1-I_{T}\left(1-\left(1-\mu_{R}(y, z)\right), 1-\left(1-\mu_{R}(y, x)\right)\right), 1-I_{T}(1-(1-\right.$ $\left.\left.\left.\mu_{R}(x, y)\right), 1-\left(1-\mu_{R}(z, y)\right)\right)\right)=\max \left(1-I_{T}\left(\mu_{R}(y, z), \mu_{R}(y, x)\right), 1-I_{T}\left(\mu_{R}(x, y), \mu_{R}(z, y)\right)\right)=$ $1-\min \left(I_{T}\left(\mu_{R}(y, z), \mu_{R}(y, x)\right), I_{T}\left(\mu_{R}(x, y), \mu_{R}(z, y)\right)\right)$.

Otherwise, $\alpha_{3}(x, y, z)=\min \left[\min \left(I_{T}\left(\mu_{R}(y, z), \mu_{R}(y, x)\right), 1-J_{S}\left(v_{R}(y, z), v_{R}(y, x)\right)\right)\right.$, $\left.\min \left(I_{T}\left(\mu_{R}(x, y), \mu_{R}(z, y)\right), 1-J_{S}\left(v_{R}(x, y), v_{R}(z, y)\right)\right)\right]=\min \left[\min \left(I_{T}\left(\mu_{R}(y, z), \mu_{R}(y, x)\right)\right.\right.$, $\left.\left.I_{T}^{1}\left(1-v_{R}(y, z), 1-v_{R}(y, x)\right)\right), \min \left(I_{T}\left(\mu_{R}(x, y), \mu_{R}(z, y)\right), I_{T}\left(1-v_{R}(x, y), 1-v_{R}(z, y)\right)\right)\right]$ $=\min \left[\min \left(I_{T}\left(\mu_{R}(y, z), \mu_{R}(y, x)\right), \quad I_{T}\left(\mu_{R}(y, z), \quad \mu_{R}(y, x)\right)\right), \min \left(I_{T}\left(\mu_{R}(x, y), \quad \mu_{R}(z, y)\right)\right.\right.$, $\left.\left.I_{T}\left(\mu_{R}(x, y), \mu_{R}(z, y)\right)\right)\right]=\min \left[I_{T}\left(\mu_{R}(y, z), \mu_{R}(y, x)\right), I_{T}\left(\mu_{R}(x, y), \mu_{R}(z, y)\right)\right]=1-\beta_{3}(x, y, z)$. Hence the result.

(iv) The proof of the equality $\beta_{4}(x, y, z)=1-\alpha_{4}(x, y, z)$ is analogous to the previous one.

(2) The proof of the last result is obvious.

Proof of Lemma 3.13. (i) $\Rightarrow$ (ii): Assume that $R$ is $\tau$-transitive on $\{x, y, z\}$ and show that (3.16). 
Since $R$ is $\tau$-transitive on $\{x, y, z\}$, then we have the following twelve inequalities:

(i) $v_{R}(x, z) \leq S\left(v_{R}(x, y), v_{R}(y, z)\right) ; \quad v_{R}(x, y) \leq S\left(v_{R}(x, z), v_{R}(z, y)\right)$;

$$
v_{R}(y, z) \leq S\left(v_{R}(y, x), v_{R}(x, z)\right)
$$

(ii) $v_{R}(z, x) \leq S\left(v_{R}(z, y), v_{R}(y, x)\right) ; \quad v_{R}(z, y) \leq S\left(v_{R}(z, x), v_{R}(x, y)\right)$;

$$
v_{R}(y, x) \leq S\left(v_{R}(y, z), v_{R}(z, x)\right)
$$

(iii) $\mu_{R}(x, z) \geq T\left(\mu_{R}(x, y), \mu_{R}(y, z)\right) ; \quad \mu_{R}(x, y) \geq T\left(\mu_{R}(x, z), \mu_{R}(z, y)\right)$;

$$
\mu_{R}(y, z) \geq T\left(\mu_{R}(y, x), \mu_{R}(x, z)\right)
$$

$$
\begin{gathered}
\mu_{R}(z, x) \geq T\left(\mu_{R}(z, y), \mu_{R}(y, x)\right) ; \quad \mu_{R}(z, y) \geq T\left(\mu_{R}(z, x), \mu_{R}(x, y)\right) \\
\mu_{R}(y, x) \geq T\left(\mu_{R}(y, z), \mu_{R}(z, x)\right) .
\end{gathered}
$$

Let us show that $\mu_{R}(x, z) \in\left[\alpha_{2}(x, y, z), \alpha_{4}(x, y, z)\right]$.

By definition of $I_{T}$, the two first inequalities of (iii) of (A.10) imply $\alpha_{2}(x, y, z)=$ $T\left(\mu_{R}(x, y), \mu_{R}(y, z)\right) \leq \mu_{R}(x, z) \leq I_{T}\left(\mu_{R}(z, y), \mu_{R}(x, y)\right)$. And the second inequalities of (i) give $J_{S}\left(v_{R}(z, y), v_{R}(x, y)\right) \leq v_{R}(x, z) \leq 1-\mu_{R}(x, z)$, that is, $\mu_{R}(x, z) \leq 1-J_{S}\left(v_{R}(z, y), v_{R}(x, y)\right)$. Thus, $\alpha_{2}(x, y, z) \leq \mu_{R}(x, z) \leq \min \left(I_{T}\left(\mu_{R}(z, y), \mu_{R}(x, y)\right), 1-J_{S}\left(v_{R}(z, y), v_{R}(x, y)\right)\right)$.

Otherwise, the third inequality of (iii) gives $\mu_{R}(x, z) \leq I_{T}\left(\mu_{R}(y, x), \mu_{R}(y, z)\right)$. The third inequality of (i) gives $J_{S}\left(v_{R}(y, x), v_{R}(y, z)\right) \leq v_{R}(x, z) \leq 1-\mu_{R}(x, z)$. Thus, $\mu_{R}(x, z) \leq$ $\min \left(I_{T}\left(\mu_{R}(y, x), \mu_{R}(y, z)\right), 1-J_{S}\left(v_{R}(y, x), v_{R}(y, z)\right)\right)$. Hence the result.

With the two first inequalities of (iv) of (A.10), the second inequality of (ii) of (A.10), the third inequality of (iv) of (A.10), and the third inequality of (ii) of (A.10), we show analogously that $\mu_{R}(z, x) \in\left[\alpha_{1}(x, y, z), \alpha_{3}(x, y, z)\right]$.

With the two first inequalities of (i) of (A.10) and the third inequality of (i) of (A.10), we show analogously that $v_{R}(x, z) \in\left[\beta_{4}(x, y, z), \beta_{2}(x, y, z)\right]$.

With the two first inequalities of (ii) of (A.10) and the third inequality of (ii) of (A.10), we show analogously that $v_{R}(z, x) \in\left[\beta_{3}(x, y, z), \beta_{1}(x, y, z)\right]$. Hence the result.

(ii) $\Rightarrow($ i): Assume (3.16), and let us show that $R$ is $\tau$-transitive on $\{x, y, z\}$. That is to show the twelve inequalities of (A.10).

The assertion $\mu_{R}(z, x) \in\left[\alpha_{1}(x, y, z), \alpha_{3}(x, y, z)\right]$ implies $\alpha_{1}(x, y, z)=$ $T\left(\mu_{R}(z, y), \mu_{R}(y, x)\right) \leq \mu_{R}(z, x), \mu_{R}(z, x) \leq I_{T}\left(\mu_{R}(y, z), \mu_{R}(y, x)\right)$ and $\mu_{R}(z, x) \leq$ $I_{T}\left(\mu_{R}(x, y), \mu_{R}(z, y)\right)$. Thus, the second result of Proposition 2.1 and the last inequalities imply $\mu_{R}(z, y) \geq T\left(\mu_{R}(z, x), \mu_{R}(x, y)\right)$ and $\mu_{R}(y, x) \geq T\left(\mu_{R}(y, z), \mu_{R}(z, x)\right)$. Hence (iv) of (A.10).

Analogously, the assertion $\mu_{R}(x, z) \in\left[\alpha_{2}(x, y, z), \alpha_{4}(x, y, z)\right]$ and the second result of Proposition 2.1 imply (iii) of (A.10); the assertion $v_{R}(x, z) \in\left[\beta_{4}(x, y, z), \beta_{2}(x, y, z)\right]$ and the second result of Proposition 2.1 imply (i) of (A.10); the assertion $v_{R}(z, x) \in$ $\left[\beta_{3}(x, y, z), \beta_{1}(x, y, z)\right]$, and the second result of Proposition 2.1 imply (ii) of (A.10).

Proof of Lemma 4.2. Let $x, y \in X$.

(1) Let us show that $v_{I}(x, y)=\max \left(v_{R}(x, y), v_{R}(y, x)\right)$.

Since $R=I \cup_{2} P$ and $I$ is symmetric, thus $v_{R}(x, y)=T\left(v_{I}(x, y), v_{P}(x, y)\right)$ and $v_{R}(y, x)=$ $T\left(v_{I}(x, y), v_{P}(y, x)\right)$. We distinguish two cases. 
(i) Suppose that $\mu_{P}(x, y)>0$ or $\left(\mu_{P}(x, y)=0\right.$ and $\left.\nu_{P}(x, y)<1\right)$.

The perfect antisymmetric of $P$ implies $v_{P}(y, x)=1$. Since $T$ is a t-norm, $v_{R}(y, x)=$ $T\left(v_{I}(x, y), 1\right)=v_{I}(x, y)$. This last equality and (i) of (2.3) imply $v_{R}(x, y)=$ $T\left(v_{I}(x, y), v_{P}(x, y)\right)=T\left(v_{R}(y, x), v_{P}(x, y)\right) \leq v_{R}(y, x)=v_{I}(x, y)$. Thus, $v_{I}(x, y)=v_{R}(y, x)=$ $\max \left[v_{R}(x, y), v_{R}(y, x)\right]$.

(ii) Suppose that $\mu_{P}(x, y)=0$ and $v_{P}(x, y)=1$.

Since $T$ is a t-norm and $I$ is symmetric, $v_{R}(x, y)=T\left(v_{I}(x, y), v_{P}(x, y)\right)=T\left(v_{I}(x, y), 1\right)=$ $v_{I}(x, y)$. This equality and (i) of (2.3) imply $v_{R}(y, x)=T\left(v_{I}(x, y), v_{P}(y, x)\right) \leq v_{I}(x, y)=$ $v_{R}(x, y)$. Thus, $v_{I}(x, y)=v_{R}(x, y)=\max \left[v_{R}(x, y), v_{R}(y, x)\right]$.

The proof of the equality $\mu_{I}(x, y)=\min \left(\mu_{R}(x, y), \mu_{R}(y, x)\right)$ is similar to the previous one.

(2) Let us show that $v_{R}(x, y) \geq v_{R}(y, x) \Leftrightarrow v_{P}(x, y)=1$.

Since $R=I \cup_{2} P$, thus $v_{R}(x, y)=T\left(v_{I}(x, y), v_{P}(x, y)\right)$ and $v_{R}(y, x)=T\left(v_{I}(y, x), v_{P}(y, x)\right)$.

$(\Rightarrow)$ : Assume to the contrary that $v_{R}(x, y) \geq v_{R}(y, x)$ and $v_{P}(x, y)<1$.

Since $P$ is perfect antisymmetric and $v_{P}(x, y)<1$, we have $v_{P}(y, x)=1$. Thus $v_{R}(y, x)=$ $T\left(v_{I}(y, x), v_{P}(y, x)\right)=v_{I}(y, x)$. Since $v_{R}(x, y) \geq v_{R}(y, x)$, the previous result gives $v_{I}(x, y)=$ $\max \left(v_{R}(x, y), v_{R}(y, x)\right)=v_{R}(x, y)$. Since $I$ is symmetric, the two previous equalities imply $v_{R}(x, y)=v_{R}(y, x)$. The $\pi$-symmetric of $R$ and the previous equality imply $\mu_{R}(x, y)=$ $\mu_{R}(y, x)$. Thus, since $P$ is simple, the two previous equalities imply $v_{P}(x, y)=v_{P}(y, x)=1$, which contradicts the hypothesis $v_{P}(x, y)<1$. Finally, $v_{R}(x, y) \geq v_{R}(y, x) \Rightarrow v_{P}(x, y)=1$.

$(\Leftarrow)$ : The proof of converse is obvious.

The proof of the equivalence $\mu_{R}(x, y) \leq \mu_{R}(y, x) \Leftrightarrow \mu_{P}(x, y)=0$ is analogous to the previous one.

Proof of Lemma 4.4. Suppose that $\mu_{R}(x, y)>\mu_{R}(y, x)$ and $v_{R}(x, y)<v_{R}(y, x)$.

(i) Consider the function $f$ defined over $[0,1]$ by $f(t)=S\left(t, \mu_{R}(y, x)\right)$, thus $f(0)=$ $\mu_{R}(y, x)$ and $f(1)=1$. $f$ is continuous and monotone because $S$ is continuous and monotone. Then $f$ takes all values between $\mu_{R}(y, x)$ and 1 . In particular, $f$ has the value $\mu_{R}(x, y)$ for $\mu_{R}(x, y)>\mu_{R}(y, x)$. Thus (4.9) $\left(E_{1}\right)$ has at least a solution.

By considering the function $g$ defined over $[0,1]$ by $g(t)=T\left(t, v_{R}(y, x)\right)$, we analogously show that $(4.9)\left(E_{2}\right)$ has at least a solution.

(ii) Let us show that each solution of $(4.9)\left(E_{1}\right)$ is strictly positive. Let $t_{1}$ be a solution of (4.9) $\left(E_{1}\right)$.

Assume to the contrary that $t_{1}=0$. Thus, $\mu_{R}(x, y)=S\left(\mu_{R}(y, x), t_{1}\right)=S\left(\mu_{R}(y, x), 0\right)=\mu_{R}(y, x)$, that is, $\mu_{R}(x, y)=\mu_{R}(y, x)$ which contradicts $\mu_{R}(x, y)>\mu_{R}(y, x)$. And we have $t_{1}>0$.

Let us show that each solution of $(4.9)\left(E_{2}\right)$ is least than 1. Let $t_{2}$ be a solution of $(4.9)\left(E_{2}\right)$. Assume to the contrary that $t_{2}=1$. Thus, $v_{R}(x, y)=T\left(v_{R}(y, x), t_{2}\right)=$ $T\left(v_{R}(y, x), 1\right)=v_{R}(y, x)$, that is, $v_{R}(x, y)=v_{R}(y, x)$ which contradicts $v_{R}(x, y)<v_{R}(y, x)$. And we have $t_{2}<1$. 
(iii) Assume that 2 satisfies condition G. We can remark by (2) of Proposition 2.1 that $S\left(\mu_{R}(y, x), J_{S}\left(\mu_{R}(y, x), \mu_{R}(x, y)\right)\right)=\mu_{R}(x, y)$ and $T\left(v_{R}(y, x), I_{T}\left(v_{R}(y, x)\right.\right.$, $\left.\left.v_{R}(x, y)\right)\right)=v_{R}(x, y)$. Since $R$ is $\pi$-symmetric, $\mu_{R}(x, y)+v_{R}(x, y)=\mu_{R}(y, x)+$ $v_{R}(y, x)$. Because 2 satisfies condition $G$, then $\mu_{R}(x, y)>\mu_{R}(y, x), v_{R}(x, y)<$ $v_{R}(y, x)$, and the previous equality imply $J_{S}\left(\mu_{R}(y, x), \mu_{R}(x, y)\right)+I_{T}\left(v_{R}(y, x)\right.$, $\left.v_{R}(x, y)\right) \leq 1$. Hence $\left(J_{S}\left(\mu_{R}(y, x), \mu_{R}(x, y)\right), I_{T}\left(v_{R}(y, x), v_{R}(x, y)\right)\right)$ is a solution of (4.8).

(iv) Assume that 2 satisfies condition $G$, and let us show that $J_{S}\left(\mu_{R}(y, x), \mu_{R}(x, y)\right)$ is the lowest solution for $(4.9)\left(E_{1}\right)$.

Consider $t_{1}$ another solution of $(4.9)\left(E_{1}\right)$. Thus we have $S\left(t_{1}, \mu_{R}(y, x)\right)=\mu_{R}(x, y)$ which implies $t_{1} \in\left\{t \in[0,1], S\left(t, \mu_{R}(y, x)\right) \geq \mu_{R}(x, y)\right\}$. Since $J_{S}\left(\mu_{R}(y, x), \mu_{R}(x, y)\right)=\min \{t \in$ $\left.[0,1], S\left(t, \mu_{R}(y, x)\right) \geq \mu_{R}(x, y)\right\}$, we deduce that $J_{S}\left(\mu_{R}(y, x), \mu_{R}(x, y)\right) \leq t_{1}$.

We analogously show that $I_{T}\left(v_{R}(y, x), v_{R}(x, y)\right)$ is the upper solution for $(4.9)\left(E_{2}\right)$.

(v) If $2=(\max , \min )$, we easily show that $\left(J_{S}\left(\mu_{R}(y, x), \mu_{R}(x, y)\right), I_{T}\left(v_{R}(y, x), v_{R}(x, y)\right)\right)$ is the unique solution of (4.8).

Suppose that $2=(S, T)$ is a strict t-conorm on $L^{*}$ satisfying condition $G$.

The previous functions $f$ and $g$ defined in (i) are, respectively, the bijections from $[0,1]$ to $\left[\mu_{R}(y, x), 1\right]$ and from $[0,1]$ to $\left[0, v_{R}(y, x)\right]$. We easily show that $\left(t_{1}, t_{2}\right)=$ $\left(J_{S}\left(\mu_{R}(y, x), \mu_{R}(x, y)\right), I_{T}\left(v_{R}(y, x), v_{R}(x, y)\right)\right)$ is the unique solution of (4.8).

Proof of Theorem 4.5. (1) $\Rightarrow(2)$ : Lemma 4.2 implies (i).

Let us show (ii). $\forall x, y \in X$, suppose that $\mu_{R}(x, y)>\mu_{R}(y, x)$ and $v_{R}(x, y)<v_{R}(y, x)$.

(iii) of Lemma 4.4 implies that (4.8) has at least one solution. Set $\left(c_{x y}, g_{x y}\right) \in L^{*}$ one of these solutions. Thus $c_{x y}$ is a solution of $(4.9)\left(E_{1}\right)$, and $g_{x y}$ is a solution of $(4.9)\left(E_{2}\right)$. With (ii) of Lemma 4.4, we have $c_{x y}>0$ and $g_{x y}<1$. Since the equality $R=I \cup_{2} P$ is equivalent to equation $\left(\mu_{R}(x, y), v_{R}(x, y)\right)=\partial\left[\left(\mu_{R}(y, x), v_{R}(y, x)\right),\left(\mu_{P}(x, y), v_{P}(x, y)\right)\right]$, hence $\mu_{P}(x, y)=c_{x y}$ and $v_{P}(x, y)=g_{x y}$.

For the case where $\mu_{R}(x, y) \leq \mu_{R}(y, x)$ and $v_{R}(x, y) \geq v_{R}(y, x)$, we easily show that Lemma 4.2 implies that $\mu_{P}(x, y)=0$ and $v_{P}(x, y)=1$.

(2) $\Rightarrow(1)$ : Let $x, y \in X$.

(i) implies that $\mu_{I}(x, y)=\mu_{I}(y, x)$, and $v_{I}(x, y)=v_{I}(y, x)$ which show that $I$ is symmetric.

Let us show that $R=I \cup_{2} P$, that is, $S\left(\mu_{I}(x, y), \mu_{P}(x, y)\right)=\mu_{R}(x, y)$ and $T\left(v_{I}(x, y), v_{P}(x, y)\right)=$ $v_{R}(x, y)$. Since $R$ is $\pi$-symmetric, we distinguish two cases.

(a) If $\mu_{R}(x, y) \leq \mu_{R}(y, x)$ and $v_{R}(x, y) \geq v_{R}(y, x)$, thus (i) implies $\mu_{I}(x, y)=\mu_{R}(x, y)$, and $v_{I}(x, y)=v_{R}(x, y)$, and the definition of $P$ gives $\mu_{P}(x, y)=0$ and $v_{P}(x, y)=1$. We have $S\left(\mu_{I}(x, y), \mu_{P}(x, y)\right)=S\left(\mu_{R}(x, y), 0\right)=\mu_{R}(x, y)$ and $T\left(v_{I}(x, y), v_{P}(x, y)\right)=$ $T\left(v_{R}(x, y), 1\right)=v_{R}(x, y)$.

(b) If $\mu_{R}(x, y)>\mu_{R}(y, x)$ and $v_{R}(x, y)<v_{R}(y, x)$, thus (ii) implies that $\mu_{P}(x, y)$ is a solution of $(4.9)\left(E_{1}\right)$, and $v_{P}(x, y)$ is a solution of $(4.9)\left(E_{2}\right)$; we have $S\left(\mu_{R}(y, x), \mu_{P}(x, y)\right)=\mu_{R}(x, y)$ and $T\left(v_{R}(y, x), v_{P}(x, y)\right)=v_{R}(x, y)$. Furthermore, as $\mu_{R}(x, y)>\mu_{R}(y, x)$, and $v_{R}(x, y)<v_{R}(y, x)$, (i) implies $\mu_{I}(x, y)=\mu_{R}(y, x)$ and $v_{I}(x, y)=v_{R}(y, x)$. Then $S\left(\mu_{I}(x, y), \mu_{P}(x, y)\right)=S\left(\mu_{R}(y, x), \mu_{P}(x, y)\right)=\mu_{R}(x, y)$ and $T\left(v_{I}(x, y), v_{P}(x, y)\right)=T\left(v_{R}(y, x), v_{P}(x, y)\right)=v_{R}(x, y)$. 
It is easy to show that $\mathrm{P}$ is simple and $P$ is perfect antisymmetric.

Proof of Corollary 4.7. The proof of this corollary is deduced from Theorem 4.5 and the last result of Lemma 4.4 .

Proof of Corollary 4.8. The proof is deduced from Lemma 4.2, Theorem 4.5, and the last result of Proposition 3.9.

Proof of Proposition 5.5. Let $x, y, z \in X$. Since $R$ is strongly complete, Corollary 3.11 implies $\alpha_{3}(x, y, z)<\alpha_{2}(x, y, z)$ and $\beta_{2}(x, y, z)<\beta_{3}(x, y, z)$.

Proof of Theorem 5.6. As $R$ is $\pi$-symmetric, (i) of (5.3) and (ii) of (5.3) are equivalent. Therefore, to show (5.3) means to show (i) of (5.3) or (ii) of (5.3).

$(\Rightarrow)$ : We distinguish two cases.

(1) Suppose that $R$ satisfies condition $C_{1}^{\mu_{R}}$, and let us show that $P$ is pos-transitive. We show (i) of (5.3). Let $x, y, z \in X$ such that $\mu_{R}(x, y)>\mu_{R}(y, x)$ and $\mu_{R}(y, z)>$ $\mu_{R}(z, y)$. Let us show that $\mu_{R}(x, z)>\mu_{R}(z, x)$. Since $R$ is $\tau$-transitive on $\{x, y, z\}$, Lemma 3.13 implies

$$
\begin{aligned}
& \mu_{R}(x, z) \in\left[\alpha_{2}(x, y, z), \alpha_{4}(x, y, z)\right], \\
& \mu_{R}(z, x) \in\left[\alpha_{1}(x, y, z), \alpha_{3}(x, y, z)\right] .
\end{aligned}
$$

We distinguish two cases.

(a) Suppose that $\alpha_{3}(x, y, z)<\alpha_{2}(x, y, z)$ or $\mu_{R}(z, x)<\alpha_{2}(x, y, z)<\alpha_{3}(x, y, z)$ or $\alpha_{2}(x, y, z)<\alpha_{3}(x, y, z)<\mu_{R}(x, z)$; thus (A.11) implies $\mu_{R}(x, z)>\mu_{R}(z, x)$.

(b) Suppose that $\alpha_{2}(x, y, z) \leq \alpha_{3}(x, y, z)$ and $\mu_{R}(z, x), \mu_{R}(x, z) \in\left[\alpha_{2}(x, y, z)\right.$, $\left.\alpha_{3}(x, y, z)\right]$; thus, since $\mu_{R}(x, y)>\mu_{R}(y, x), \mu_{R}(y, z)>\mu_{R}(z, y)$ and $R$ satisfies condition $C_{1}^{\mu_{R}}$, we deduce that $\mu_{R}(x, z)>\mu_{R}(z, x)$.

(2) With Lemma 4.2, the proof is analogous to the previous one.

$(\Leftarrow)$ Suppose that $P$ is pos-transitive, and let us show that $R$ satisfies $C_{1}^{\mu_{R}}$ or $C_{1}^{\nu_{R}}$.

Since $P$ is pos-transitive, we have (5.3), that is, (i) of (5.3) or (ii) of (5.3). We distinguish two cases.

(a) Suppose (ii) of (5.3), and let us show that $R$ satisfies $C_{1}^{\nu_{R}}$. Suppose that $R$ verifies

$$
\begin{aligned}
& v_{R}(y, x)>v_{R}(x, y), \\
& v_{R}(z, y)>v_{R}(y, z),
\end{aligned}
$$

and $v_{R}(x, z), v_{R}(z, x) \in\left[\beta_{3}(x, y, z), \beta_{2}(x, y, z)\right]$. Let us show that $v_{R}(x, z)<v_{R}(z, x)$. Since $R$ satisfies

$$
\begin{aligned}
& v_{R}(y, x)>v_{R}(x, y), \\
& v_{R}(z, y)>v_{R}(y, z),
\end{aligned}
$$

the two previous inequalities and (ii) of (5.3) imply $v_{R}(x, z)<v_{R}(z, x)$.

(b) With (i) of (5.3), we show analogously that $R$ satisfies $C_{1}^{\mu_{R}}$. 
Proof of Corollary 5.7. Since $R$ is strongly complete, then Proposition 5.5 implies that $R$ satisfies condition $C_{1}^{\mu_{R}}$. Hence, Theorem 5.6 implies that $P$ is pos-transitive.

Proof of Lemma 5.8. To establish (5.15) is equivalent to establish (5.16).

We remark that as $R$ is $\pi$-symmetric, (i) of (5.16) and (ii) of (5.16) are equivalent.

$(1) \Rightarrow(2)$ : We distinguish two cases.

(1) Suppose that $R$ satisfies condition $C_{2}^{\mu_{R}}$, and let us show (5.16). We show (i) of (5.16). Let $x, y, z \in X$ such that

$$
\left\{\begin{array} { l } 
{ \mu _ { R } ( y , x ) = \mu _ { R } ( x , y ) } \\
{ \mu _ { R } ( z , y ) < \mu _ { R } ( y , z ) }
\end{array} \text { or } \quad \left\{\begin{array}{l}
\mu_{R}(y, x)<\mu_{R}(x, y) \\
\mu_{R}(z, y)=\mu_{R}(y, z) .
\end{array}\right.\right.
$$

Let us show that $\mu_{R}(x, z)>\mu_{R}(z, x)$. Since $R$ is $\tau$-transitive on $\{x, y, z\}$, Lemma 3.13 implies (A.11). We distinguish two cases.

(a) Suppose that $\alpha_{3}(x, y, z)<\alpha_{2}(x, y, z)$ or $\mu_{R}(z, x)<\alpha_{2}(x, y, z)<\alpha_{3}(x, y, z)$ or $\alpha_{2}(x, y, z)<\alpha_{3}(x, y, z)<\mu_{R}(x, z)$; thus (A.11) implies $\mu_{R}(x, z)>\mu_{R}(z, x)$.

(b) Suppose that $\alpha_{2}(x, y, z) \leq \alpha_{3}(x, y, z)$ and $\mu_{R}(z, x), \mu_{R}(x, z) \in\left[\alpha_{2}(x, y, z)\right.$, $\left.\alpha_{3}(x, y, z)\right]$. With (A.14), the condition $C_{2}^{\mu_{R}}$ implies that $\mu_{R}(x, z)>\mu_{R}(z, x)$.

(2) Suppose that $R$ satisfies condition $C_{2}^{\nu_{R}}$, and let us show (5.16). We show (ii) of (5.16). Let $x, y, z \in X$ such that

$$
\left\{\begin{array} { l } 
{ v _ { R } ( y , x ) = v _ { R } ( x , y ) } \\
{ v _ { R } ( z , y ) > v _ { R } ( y , z ) }
\end{array} \quad \text { or } \quad \left\{\begin{array}{l}
v_{R}(y, x)>v_{R}(x, y) \\
v_{R}(z, y)=v_{R}(y, z) .
\end{array}\right.\right.
$$

Let us show that $v_{R}(x, z)<v_{R}(z, x)$. Since $R$ is $\tau$-transitive on $\{x, y, z\}$, Lemma 4.2 implies

$$
\begin{aligned}
& v_{R}(x, z) \in\left[\beta_{4}(x, y, z), \beta_{2}(x, y, z)\right], \\
& v_{R}(z, x) \in\left[\beta_{3}(x, y, z), \beta_{1}(x, y, z)\right] .
\end{aligned}
$$

We distinguish two cases.

(a) Suppose that $\beta_{3}(x, y, z)>\beta_{2}(x, y, z)$ or $v_{R}(z, x)>\beta_{2}(x, y, z)>\beta_{3}(x, y, z)$ or $\beta_{2}(x, y, z)>\beta_{3}(x, y, z)>v_{R}(x, z)$; thus (A.16) implies that $v_{R}(x, z)<v_{R}(z, x)$.

(b) Suppose that $\beta_{2}(x, y, z) \geq \beta_{3}(x, y, z)$ and $v_{R}(z, x), v_{R}(x, z) \in\left[\beta_{3}(x, y, z)\right.$, $\left.\beta_{2}(x, y, z)\right]$. With (A.15), the condition $C_{2}^{\nu_{R}}$ implies that $v_{R}(x, z)<v_{R}(z, x)$.

$(2) \Rightarrow(1)$ Suppose (5.16), and let us show that $R$ satisfies $C_{2}^{\mu_{R}}$ or $C_{2}^{v_{R}}$. We distinguish two cases.

(a) Suppose (i) of (5.16), and let us show that $R$ satisfies $C_{2}^{\mu_{R}}$. Suppose (A.14), and $\mu_{R}(x, z), \mu_{R}(z, x) \in\left[\alpha_{2}(x, y, z), \alpha_{3}(x, y, z)\right]$. Let us show that $\mu_{R}(x, z)>\mu_{R}(z, x)$. With (A.14), (i) of (5.16) implies $\mu_{R}(x, z)>\mu_{R}(z, x)$.

(b) With (ii) of (5.16), we show analogously that $R$ satisfies $C_{1}^{v_{R}}$. 
Proof of Theorem 5.9. As $R$ is $\pi$-symmetric, Remark 5.2 implies that (5.4) and (5.5) are equivalent. Therefore, to show (5.4) means to show (5.4) or (5.5).

$(\Rightarrow)$ : We distinguish two cases.

(1) Suppose that $R$ satisfies condition $C_{1}^{\mu_{R}}$ and $C_{2}^{\mu_{R}}$, and let us show that $P$ is negative transitive. We show (5.4). Let $x, y, z \in X$. We distinguish three cases.

(a) Suppose

$$
\begin{aligned}
& \mu_{R}(y, x)<\mu_{R}(x, y), \\
& \mu_{R}(z, y)<\mu_{R}(y, z),
\end{aligned}
$$

and let us show that $\mu_{R}(x, z) \geq \mu_{R}(z, x)$. Since $R$ satisfies condition $C_{1}^{\mu_{R}}$, Theorem 5.6 implies that $P$ is pos-transitive. Thus,

$$
\begin{aligned}
& \mu_{R}(y, x)<\mu_{R}(x, y), \\
& \mu_{R}(z, y)<\mu_{R}(y, z),
\end{aligned}
$$

and the pos-transitivity of $P$ imply that $\mu_{R}(x, z)>\mu_{R}(z, x)$. Hence $\mu_{R}(x, z) \geq$ $\mu_{R}(z, x)$.

(b) Suppose (A.14) and let us show that $\mu_{R}(x, z) \geq \mu_{R}(z, x)$. Since $R$ satisfies condition $C_{2}^{\mu_{R}}$ and (A.14), then Lemma 5.8 implies that $\mu_{R}(x, z)>\mu_{R}(z, x)$. Hence $\mu_{R}(x, z) \geq \mu_{R}(z, x)$.

(c) Suppose that $\mu_{R}(x, y)=\mu_{R}(y, x)$ and $\mu_{R}(y, z)=\mu_{R}(z, y)$, and let us show that $\mu_{R}(x, z) \geq \mu_{R}(z, x)$. Assume to the contrary that $\mu_{R}(x, z)<\mu_{R}(z, x)$. Thus, $\mu_{R}(x, y)=\mu_{R}(y, x)$ and $\mu_{R}(x, z)<\mu_{R}(z, x)$, that is,

$$
\begin{aligned}
& \mu_{R}(x, z)<\mu_{R}(z, x), \\
& \mu_{R}(x, y)=\mu_{R}(y, x) .
\end{aligned}
$$

Since $R$ satisfies condition $C_{2}^{\mu_{R}}$ and

$$
\begin{aligned}
& \mu_{R}(x, z)<\mu_{R}(z, x), \\
& \mu_{R}(x, y)=\mu_{R}(y, x),
\end{aligned}
$$

then Lemma 5.8 implies that $\mu_{R}(z, y)>\mu_{R}(y, z)$ which contradicts the hypothesis $\mu_{R}(y, z)=\mu_{R}(z, y)$.

(2) Suppose that $R$ satisfies condition $C_{1}^{\nu_{R}}$ and $C_{2}^{v_{R}}$, and let us show that $P$ is negative transitive. We show (5.5). Let $x, y, z \in X$. We distinguish three cases. 
(a) Suppose that $R$ verifies

$$
\begin{aligned}
& v_{R}(y, x)>v_{R}(x, y), \\
& v_{R}(z, y)>v_{R}(y, z),
\end{aligned}
$$

and let us show that $v_{R}(x, z) \leq v_{R}(z, x)$. Since $R$ satisfies condition $C_{1}^{v_{R}}$, Theorem 5.6 implies that $P$ is pos-transitive. Thus,

$$
\begin{aligned}
& v_{R}(y, x)>v_{R}(x, y), \\
& v_{R}(z, y)>v_{R}(y, z),
\end{aligned}
$$

and the pos-transitivity of $P$ imply that $v_{R}(x, z)<v_{R}(z, x)$. Hence $v_{R}(x, z) \leq$ $v_{R}(z, x)$.

(b) Suppose (A.15), and let us show that $v_{R}(x, z) \leq v_{R}(z, x)$. Since $R$ satisfies condition $C_{2}^{v_{R}}$ and (A.15), then Lemma 5.8 implies that $v_{R}(x, z)<v_{R}(z, x)$. Hence $v_{R}(x, z) \leq v_{R}(z, x)$.

(c) Suppose that $v_{R}(x, y)=v_{R}(y, x)$ and $v_{R}(y, z)=v_{R}(z, y)$; and let us show that $v_{R}(x, z) \leq v_{R}(z, x)$. Assume to the contrary that $v_{R}(x, z)>v_{R}(z, x)$. Thus,

$$
\begin{gathered}
v_{R}(x, z)>v_{R}(z, x), \\
v_{R}(x, y)=v_{R}(y, x) .
\end{gathered}
$$

Since $R$ satisfies condition $C_{2}^{v_{R}}$ and

$$
\begin{gathered}
v_{R}(x, z)>v_{R}(z, x), \\
v_{R}(x, y)=v_{R}(y, x),
\end{gathered}
$$

then Lemma 5.8 implies $v_{R}(z, y)<v_{R}(y, z)$ which contradicts hypothesis $v_{R}(y, z)=v_{R}(z, y)$.

$(\Leftarrow)$ Suppose that $P$ is negative transitive, and let us show that $R$ satisfies $\left(C_{1}^{\mu_{R}}\right.$ and $\left.C_{2}^{\mu_{R}}\right)$ or $\left(C_{1}^{\nu_{R}}\right.$ and $\left.C_{2}^{\nu_{R}}\right)$.

Since $P$ is negative transitive, we have (5.4) and (5.5), that is, (5.4) or (5.5) as $R$ is $\pi$ symmetric. We distinguish two cases.

(i) Suppose (5.4), and let us show that $R$ satisfies $C_{1}^{\mu_{R}}$ and $C_{2}^{\mu_{R}}$. Suppose that $\mu_{R}(x, z), \mu_{R}(z, x) \in\left[\alpha_{2}(x, y, z), \alpha_{3}(x, y, z)\right]$ and (3.9). Let us show that $\mu_{R}(x, z)>$ $\mu_{R}(z, x)$. In fact, (3.9) and (5.4) imply $\mu_{R}(x, z) \geq \mu_{R}(z, x)$. Assume to the contrary that $\mu_{R}(x, z)=\mu_{R}(z, x)$. With (3.9), we distinguish two cases.

(a) If $\mu_{R}(x, y)>\mu_{R}(y, x)$ and $\mu_{R}(y, z) \geq \mu_{R}(z, y)$; thus we have $\mu_{R}(x, z)=\mu_{R}(z, x)$ and $\mu_{R}(y, z) \geq \mu_{R}(z, y)$. Hence the negative transitivity of $P$ implies $\mu_{R}(y, x) \geq$ $\mu_{R}(x, y)$ which contradicts the hypothesis $\mu_{R}(x, y)>\mu_{R}(y, x)$. 
(b) If $\mu_{R}(x, y)=\mu_{R}(y, x)$ and $\mu_{R}(y, z)>\mu_{R}(z, y)$; thus we have $\mu_{R}(x, z)=\mu_{R}(z, x)$ and $\mu_{R}(y, z)=\mu_{R}(z, y)$. Hence the negative transitivity of $P$ implies $\mu_{R}(z, y) \geq$ $\mu_{R}(y, z)$ which contradicts the hypothesis $\mu_{R}(y, z)>\mu_{R}(z, y)$.

(ii) Suppose (5.5) and let us show that $R$ satisfies $C_{1}^{v_{R}}$ and $C_{2}^{v_{R}}$. Suppose (3.11) and $v_{R}(x, z), v_{R}(z, x) \in\left[\beta_{3}(x, y, z), \beta_{2}(x, y, z)\right]$. Let us show that $v_{R}(x, z)<v_{R}(z, x)$. In fact, (3.11) and (5.5) imply $v_{R}(x, z) \leq v_{R}(z, x)$. Assume to the contrary that $v_{R}(x, z)=v_{R}(z, x)$. With (3.11), we distinguish two cases.

(a) If $v_{R}(x, y)<v_{R}(y, x)$ and $v_{R}(y, z) \leq v_{R}(z, y)$; thus we have $v_{R}(x, z)=v_{R}(z, x)$ and $v_{R}(y, z) \leq v_{R}(z, y)$. Hence the negative transitivity of $P$ implies $v_{R}(y, x) \leq$ $v_{R}(x, y)$ which contradicts the hypothesis $v_{R}(x, y)<v_{R}(y, x)$.

(b) If $v_{R}(x, y)=v_{R}(y, x)$ and $v_{R}(y, z)<v_{R}(z, y)$; thus we have $v_{R}(x, z)=v_{R}(z, x)$ and $v_{R}(y, z)=v_{R}(z, y)$. Hence the negative transitivity of $P$ implies $v_{R}(z, y) \leq$ $v_{R}(y, z)$ which contradicts the hypothesis $v_{R}(y, z)<v_{R}(z, y)$.

Proof of Corollary 5.10. The proof is deduced from Proposition 5.5 and Theorem 5.9.

\section{Acknowledgments}

The authors are thankful to the members of Laboratoire MASS and those of CREM for their help and advice. The second author thanks French Governement and AUF (Agence Universitaire de la Francophonie). This work was achieved when he was student at Université de Caen Basse-Normandie France, under the research grants "Bourse de formation à la recherche de la Francophonie 2007-2008" and "Bourse de doctorat du SCAC-Yaoundé 2007-2008".

\section{References}

[1] L. A. Zadeh, "Fuzzy sets," Information and Computation, vol. 8, pp. 338-353, 1965.

[2] B. De Baets, E. Kerre, and B. Van de Walle, "Fuzzy preference structures and their characterization," Journal of Fuzzy Mathematics, vol. 3, no. 2, pp. 373-381, 1995.

[3] B. Dutta, "Fuzzy preferences and social choice," Mathematical Social Sciences, vol. 13, no. 3, pp. 215$229,1987$.

[4] J. Fodor and M. Roubens, Fuzzy Preference Modelling and Multicriteria Decision Support, Kluwer Academic Publishers, Dordrecht, The Netherlands, 1994.

[5] L. A. Fono, H. Gwet, and S. Fotso, "On strict lower and upper sections of weakly complete fuzzy pre-orders based on co-implication," Fuzzy Sets and Systems, vol. 159, no. 17, pp. 2240-2255, 2008.

[6] L. A. Fono and N. G. Andjiga, "Utility function of fuzzy preferences on a countable set under max-*transitivity," Social Choice and Welfare, vol. 28, no. 4, pp. 667-683, 2007.

[7] L. A. Fono and N. G. Andjiga, "Fuzzy strict preference and social choice," Fuzzy Sets and Systems, vol. 155, no. 3, pp. 372-389, 2005.

[8] L. A. Fono, Relation binaire floue et application au choix social, Thése de Doctorat, Département de Mathématiques, Faculté de Sciences, Université de Yaoundé I, Yaoundé, Cameroon, 2004.

[9] L. A. Fono and H. Gwet, "On strict lower and upper sections of fuzzy orderings," Fuzzy Sets and Systems, vol. 139, no. 3, pp. 583-599, 2003.

[10] G. Richardson, "The structure of fuzzy preferences: social choice implications," Social Choice and Welfare, vol. 15, no. 3, pp. 359-369, 1998.

[11] M. Salles, "Fuzzy Utility," in Handbook of Utility Theory, Principles, vol. 1, pp. 321-344, Kluwer Academic Publishers, Boston, Mass, USA, 1998.

[12] K. T. Atanassov, "Intuitionistic fuzzy sets," Fuzzy Sets and Systems, vol. 20, no. 1, pp. 87-96, 1986. 
[13] K. T. Atanassov and S. Stoeva, "Intuitionistic fuzzy sets," in Proceedings of the Polish Symposium on Interval and Fuzzy Mathematics, Poznan, Poland, 1983.

[14] H. Bustince, "Construction of intuitionistic fuzzy relations with predetermined properties," Fuzzy Sets and Systems, vol. 109, no. 3, pp. 379-403, 2000.

[15] H. Bustince and P. Burillo, "Structures on intuitionistic fuzzy relations," Fuzzy Sets and Systems, vol. 78, no. 3, pp. 293-303, 1996.

[16] G. Deschrijver and E. E. Kerre, “On the composition of intuitionistic fuzzy relations," Fuzzy Sets and Systems, vol. 136, no. 3, pp. 333-361, 2003.

[17] D. Dimitrov, "The Paretian liberal with intuitionistic fuzzy preferences: a result," Social Choice and Welfare, vol. 23, no. 1, pp. 149-156, 2004.

[18] D. Dimitrov, "Intuitionistic fuzzy preferences: a factorization," Advanced Studies in Contemporary Mathematics, vol. 5, no. 1, pp. 93-104, 2002.

[19] Z. Xu, "Intuitionistic preference relations and their application in group decision making," Information Sciences, vol. 177, no. 11, pp. 2363-2379, 2007.

[20] C. Cornelis, G. Deschrijver, and E. E. Kerre, "Implication in intuitionistic fuzzy and intervalvalued fuzzy set theory: construction, classification, application," International Journal of Approximate Reasoning, vol. 35, no. 1, pp. 55-95, 2004.

[21] L. A. Fono, H. Gwet, and B. Bouchon-Meunier, "Fuzzy implications operators for difference operations for fuzzy sets and cardinality-based measures of comparaison," European Journal of Operational Research, vol. 183, pp. 314-326, 2008.

[22] E. P. Klement, R. Mesiar, and E. Pap, Triangular Norms, vol. 8 of Trends in Logic-Studia Logica Library, Kluwer Academic Publishers, Dordrecht, The Netherlands, 2000.

[23] B. De Baets, "Coimplicators, the forgotten connectives," Tatra Mountains Mathematical Publications, vol. 12, pp. 229-240, 1997. 


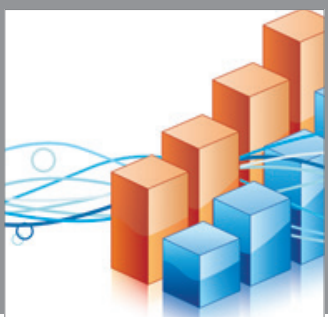

Advances in

Operations Research

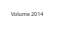

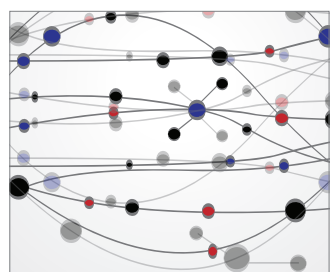

\section{The Scientific} World Journal
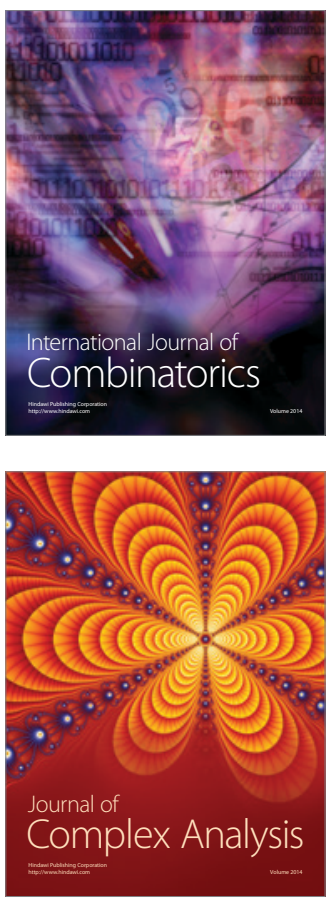

International Journal of

Mathematics and

Mathematical

Sciences
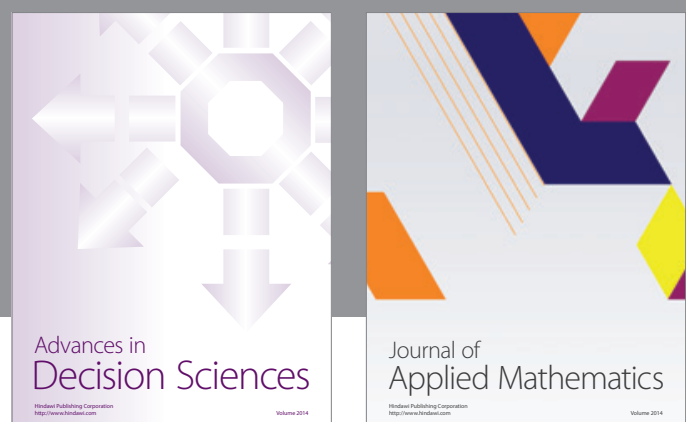

Journal of

Applied Mathematics
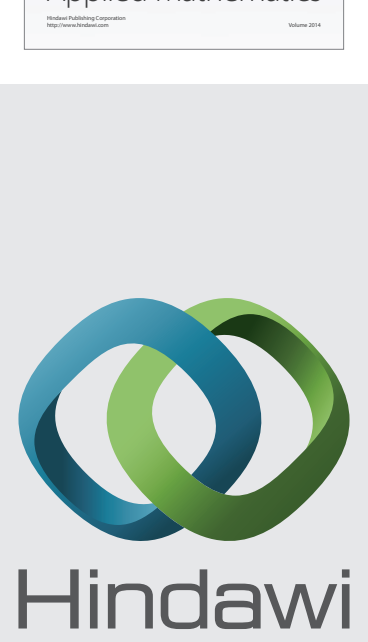

Submit your manuscripts at http://www.hindawi.com
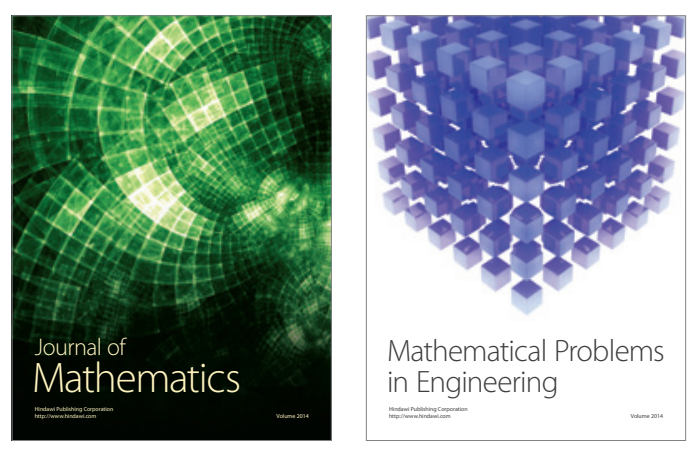

Mathematical Problems in Engineering
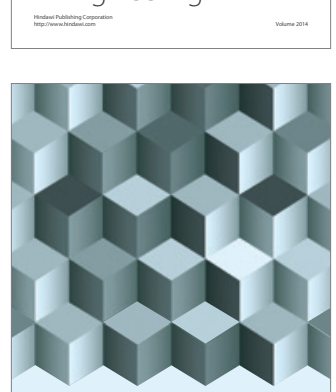

Journal of

Function Spaces
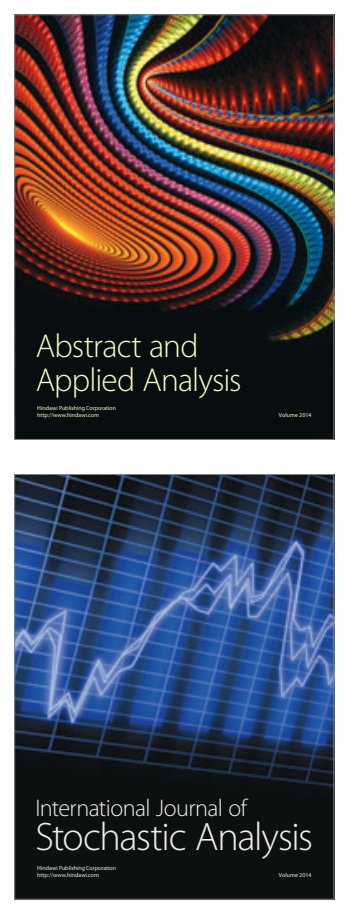

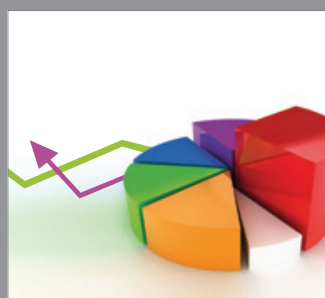

ournal of

Probability and Statistics

Promensencen
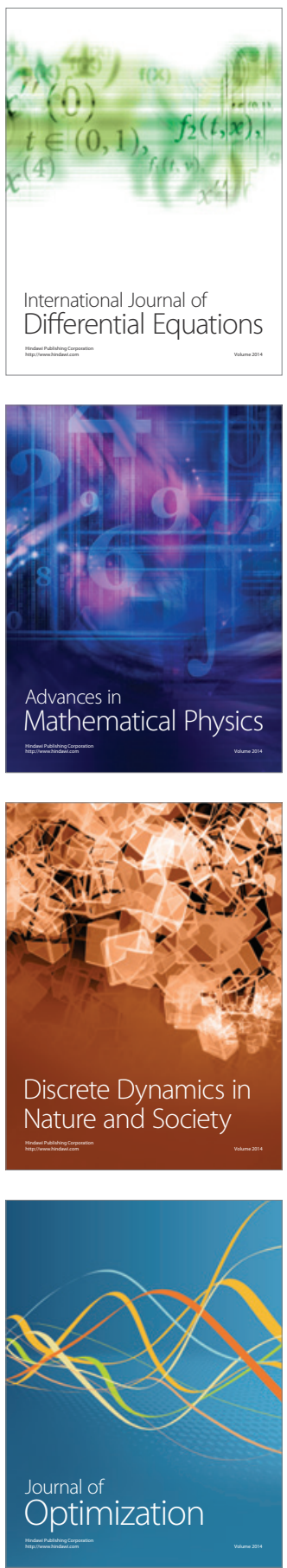